\title{
ESTRATEGIAS DE CAPTURA DE PRESAS Y SISTEMAS DE ARMAS DE LOS CAZADORES-RECOLECTORES QUE HABITARON EL HUMEDAL DEL PARANÁ INFERIOR: UNA APROXIMACIÓN EXPERIMENTAL Y ARQUEOLÓGICA.
}

\section{STRATEGIES OF PREY CAPTURE AND WEAPON SYSTEM IN HUNTER- GATHERER SOCIETIES THAT INHABITED THE LOW PARANÁ RIVER: AN EXPERIMENTAL AND ARCHAEOLOGICAL APPROACH.}

\author{
Romina Sacur Silvestre ${ }^{1}$, Natacha Buc ${ }^{2}$, Alejandro Acosta ${ }^{2}$ y Daniel \\ Loponte $^{2}$
}

\author{
${ }^{1}$ INAPL. E-mail: romisilvestre@hotmail.com \\ ${ }^{2}$ INAPL. CONICET. E-mail: romisilvestre@hotmail.com \\ ${ }^{21}$ NAPL. CONICET. E-mail: natachabuc@gmail.com \\ ${ }^{21}$ NAPL. CONICET. E-mail: acosta@retina.ar \\ ${ }^{2 I}$ NAPL. CONICET. E-mail: dashtown@gmail.com
}

Presentado el: 15/12/2011 - Aceptado 13/06/2012

\begin{abstract}
Resumen
Los conjuntos artefactuales de los sitios de cazadores-recolectores que habitaron el humedal del Paraná inferior durante el Holoceno reciente cuentan con una gran variedad de puntas líticas y óseas, destacándose entre estas últimas las puntas planas pedunculadas, las bipuntas y las puntas ahuecadas. El objetivo último de este trabajo es evaluar la relación que habrían tenido dichas piezas con los diferentes sistemas de armas y estrategias generadas para la captura de las presas. Para ello, en primer lugar se evaluaron las estructuras físicas, métricas, morfológicas y microscópicas de los distintos grupos morfológicos de puntas arqueológicas proponiendo su asociación con determinados sistemas de armas. A partir de ello, desarrollamos un programa experimental con el objetivo de evaluar la performance de cada uno bajo las condiciones esperadas.
\end{abstract}

Palabras claves: Humedal del Paraná Inferior, Sistemas de armas, Análisis funcional.

\begin{abstract}
Tool assemblages from sites of hunter-gatherers that inhabited the low Paraná wetland during late Holocene times have a great variety of lithic and bone points. Among these last ones, we highlight the presence of stemmed points, bipoints and drilled points. The main aim of this paper is to evaluate the link between these points and singular weapon systems and strategies developed for prey capture. For
\end{abstract}


this purpose, in the first place, we evaluate physic, metric, morphologic and microscopic structures of prehistoric morphological groups and suggest their association with specific weapon systems. Following that, we developed an experimental program in order to test the expected performance under those circumstances.

Keywords: Low Paraná wetland, Weapon systems, Use-wear analysis.

\section{Introducción}

El humedal del río Paraná inferior (HPI), desde el punto de vista biogeográfico, corresponde a la unidad eco-regional denominada "Delta e islas del Paraná" (Burkart et al. 2000). La misma posee una elevada productividad ambiental básicamente regulada por los pulsos de inundación del río Paraná (Bó y Málvarez 1999), y condiciones geomórficas y ecológicas establecidas luego de la última fase regresiva del nivel marino hace unos 3,5 ka años AP (Cavalotto et al. 2004; Loponte 2008).

En este trabajo nos referiremos a un sector del HPI denominado Bajíos Ribereños (Bonfils 1962) y, particularmente, a su porción meridional (BRM). La alta y concentrada oferta de recursos que presenta en general este ambiente, y otra serie de atributos relacionados con su disponibilidad, densidad y biomasa asociada, habría incentivado la formación de sistemas cazadores-recolectores con una baja movilidad asociada a una estabilidad residencial relativamente alta, siendo ambas conductas consistentes con los modelos o estrategias de lugar central o "central place foraging" (CPF) (cf. Orians y Pearson 1979; ver aplicación en Loponte 2008). Esta forma de organización se la ha vinculado con la emergencia de las denominadas sociedades cazadoras-recolectoras complejas, al igual que otras propiedades tales como la intensificación en la explotación de los recursos y la generación de sofisticados equipamientos tecnológicos a fin de maximizar su obtención y/o procesamiento (Ames 2005; Binford 2001), rasgos que también hemos identificado en nuestro caso de estudio (Acosta 2005; Loponte 2008; Loponte et al. 2006).

En los BRM, a partir de un esquema de CPF y teniendo en cuenta la distribución espacial y otros atributos de los principales recursos explotados, se estimó que los cazadoresrecolectores tuvieron un radio de predación terrestre diario de entre 4 y $6 \mathrm{~km}$, mientras que en los sectores fluviales el uso de dispositivos de navegación habría permitido rangos más extensos $(>10 \mathrm{~km})$, aumentando de este modo la capacidad de transferencia de los recursos explotados desde diferentes puntos del paisaje hacia los campamentos centrales. Esta situación también debió reducir los costos vinculados a la búsqueda y transporte de recursos, así como alentar la conformación de grupos de predación de distintas clases sexoetarias para su obtención y traslado, ya sea de manera individual o en masa ( $c f$. Loponte 2008). Asimismo, el acopio de algunos recursos habría estimulado el consumo diferido a través de su conservación y/o almacenamiento (sensu Woodburn 1980). La generación de un excedente susceptible de ser almacenado constituye una forma de optimizar los recursos en función de su abundancia y disponibilidad temporal (Binford 2001; Kelly 1995). En este sentido, creemos que dichas conductas debieron estar impulsadas en gran medida por la abundante oferta de recursos que presentan los BRM durante la estación estival; período en el que se produce la fructificación de la mayoría de las especies vegetales comestibles y aumenta de modo significativo la biomasa ictícola como resultado de los procesos migratorios de los peces de mayor valor económico (Loponte 2008). Cabe destacar que éstos junto a Blastocerus dichotomus (ciervo de los pantanos) y Ozotoceros bezoarticus (venado de 
las pampas) constituyen los recursos faunísticos que mayor cantidad de biomasa habrían aportado a la dieta. También fueron regularmente explotados los roedores Myocastor coypus (coipo) y Cavia aperea (cuis), aunque su contribución en términos de biomasa habría sido mucho menor respecto de las anteriores presas. La frecuencia taxonómica que presentan dichos taxa en los conjuntos arqueofaunísticos es consistente con su oferta natural y con sus características etoecológicas.

El registro arqueológico y las fuentes históricas disponibles para el HPI y áreas adyacentes permiten sostener que los grupos cazadores-recolectores desarrollaron diferentes sistemas de captura de las presas (cf. Acosta 2005; Buc 2010b; Loponte 2008; Musali 2010). Las estrategias implementadas habrían involucrado una gran variedad de tácticas y armas entre las que pueden incluirse el uso de bolas de boleadoras, trampas, redes, arpones, lanzas, propulsores y arcos. Estos cuatro últimos involucraban la producción de cabezales que podrían confeccionarse sobre diferentes materias primas. Por ejemplo, señala Paucke (1944 [1829] p. 160) para la zona del Chaco, entre los mocovíes "Las armas son arco de flechas, una lanza de un largo de seis varas, una porra gruesa bien labrada (...) A la par de éstas tienen una cantidad de flechas de puntas diversas y de diferentes materias". Para el mismo contexto Ambrosetti (Bathata 1893) describe el funcionamiento del sistema de lanzas de puntas de hueso separable:

"Las armas que usan los chaqueños son las flechas, lanzas y macanas. (...) Las fijas son de de varias clases, ya de hierro o de hueso, esta última es muy interesante: cortan un cuerno de ciervo o un hueso largo en forma de punta aguda, en la parte inferior lo cortan de pico de clarinete y en el medio lo agujerean, este agujero les sirve para aplicarle una cuerda larga. Este aparato va encajado en un asta de palo duro. Al clavar una pieza grande entre las costillas, sacan con fuerza el palo y como está sólo encajado, zafa, quedando la punta de hueso adentro, que como tiene la soga atada en el medio se da vuelta, quedando trancado sin poder salir entre las dos costillas; por la cuerda sujetan la víctima ya sea a mano o atándola a un árbol, rematándola en seguida" (Bathata 1893: 69-70; ver también Herberts 1998; Sánchez Labrador 1910).

En cuanto a los propulsores, Fernández de Oviedo y Valdés (1944[1526]) menciona la utilización de estos artefactos para los timbús y chanás de las costas del Río de la Plata; hecho corroborado por la identificación de ganchos/tacos de propulsor en los sitios arqueológicos del área (Buc 2010b; Loponte 2008; Lothrop 1932; Torres 1911).

Todo ello debió ofrecer distintas posibilidades para la captura de presas, ampliadas aún más si consideramos la complementación con el uso de canoas (Acosta 2005; Loponte 2008; Musali 2010). Por ejemplo, las bolas de boleadoras y los propulsores seguramente constituyeron sistemas diseñados para actuar en ambientes relativamente abiertos y debieron estar preferentemente orientados hacia presas terrestres de mediano y gran porte como habrían sido O. bezoarticus, B. dichotomus o incluso Lama guanicoe (cf. Loponte 2008). De hecho, estos sistemas están bien representados entre los grupos llamados "querandíes" habitantes de la Pampa Ondulada (Azara 1943[1847]; Schmidel 1988[1567]). En cambio, el arco y flecha brinda una mayor versatilidad, ya que puede utilizarse en sectores terrestres abiertos, pero también constituye un sistema de gran precisión para los ambientes de bosque y en la captura de peces, embarcado o desde tierra. Lo mismo podemos decir para las lanzas, cuya diferencia radica en la menor precisión del sistema (cf. Churchill 1993; Loponte 2008). Los arpones, finalmente, tienen un rango de acción mucho más definido, dado que son efectivos para pescar determinadas especies que suelen estar cerca de la superficie y/o en cursos de agua poco profundos (Acosta 2005; Loponte 2008; Musali y Buc 20011). 
El uso de una variada gama de estrategias habría posibilitado minimizar el riesgo de pérdida de las presas (sensu Bousman 1993). En tal sentido, uno de los principales objetivos de este trabajo es explorar algunas propiedades y la efectividad de los sistemas de armas utilizados, particularmente aquellos vinculados al uso de los cabezales óseos y líticos. No se incluye aquí el grupo de arpones, ya que fue objeto de estudio en otros trabajos (Buc 2007; Musali y Buc 20011).

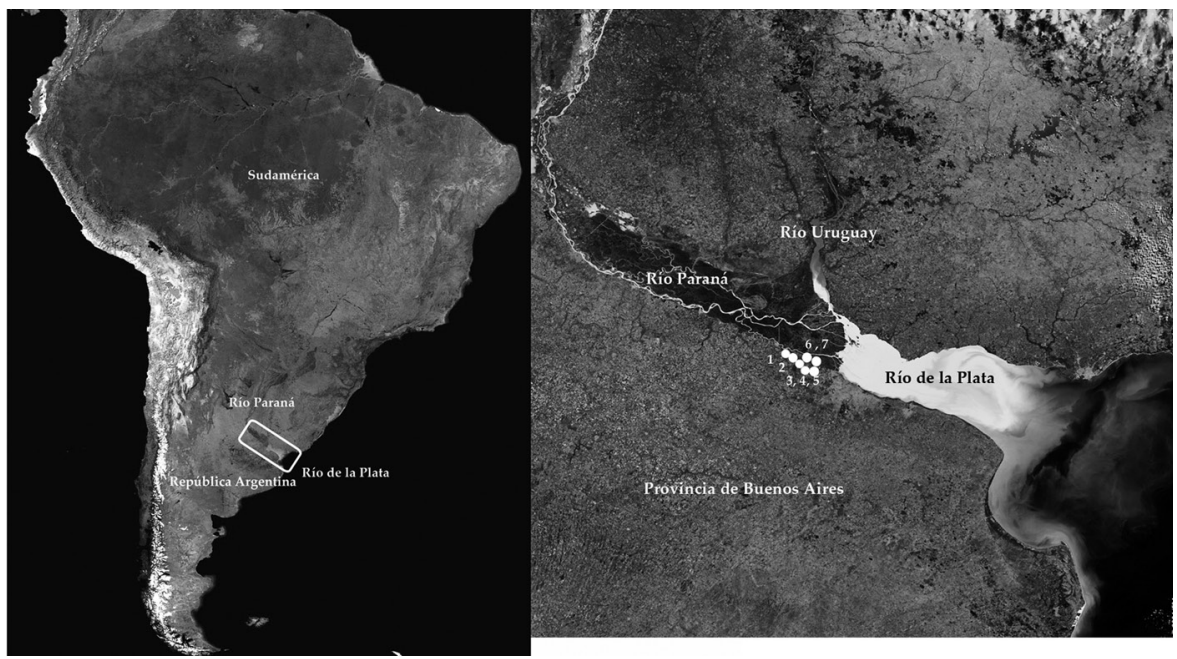

Figura 1. Izquierda: Localización del HPI. Derecha: detalle del HPI con la ubicación de los sitios analizados. 1: Las Vizcacheras, 2: Anahí, 3: Guazunambí, 4: La Bellaca Sitio 1, 5: La Bellaca Sitio 2, 6: Garín, 7: Punta Canal.

\section{Materiales y métodos}

Los cabezales líticos y óseos que aquí se discuten provienen de siete depósitos arqueológicos: Anahí, Garín, Guazunambí, La Bellaca 1, La Bellaca 2, Las Vizcacheras y Punta Canal. Todos estos sitios se encuentran localizados en los BRM (Figura 1) y se ubican dentro de los últimos 1000 años C14 AP (cf. Arrizurieta et al. 2010; Loponte 2008). Los análisis efectuados y las diferentes líneas de evidencias indican que todos los depósitos mencionados pueden ser funcionalmente atribuidos a la categoría de sitios de actividades múltiples (Acosta 2005; Loponte 2008).

A pesar de que en los BRM no existen afloramientos rocosos locales, en casi todos los sitios se han reconocido artefactos elaborados sobre distintas materias primas líticas, cuyo abastecimiento habría implicado activas redes de intercambio tanto dentro del HPI como con otras regiones distantes (Loponte 2008; Silvestre 2010). Debido a esta característica, las puntas de proyectil y otros artefactos líticos presentan una frecuencia relativamente baja en relación a los numerosos instrumentos óseos recuperados, cuyo grupo morfológico dominante corresponde al de las denominadas "puntas". Este grupo incluye principalmente cabezales de arpones, puntas planas pedunculadas, puntas ahuecadas y bipuntas (Pérez Jimeno y Buc 2009). Si bien sabemos la dificultad de asociar morfologías de cabezales con sistemas de armas (ver por ejemplo una de las primeras discusiones en Browne 1938; Kidder 1938), el registro arqueológico y las crónicas históricas sugieren la posibilidad de al menos tres sistemas de captura, más allá de los arpones que no discutiremos aquí: propulsores, lanzas y arco. 
Las puntas líticas y las puntas óseas planas pedunculadas presentan rasgos asimilables a la categoría de puntas de proyectil. En cuanto a las puntas ahuecadas y las bipuntas es importante mencionar que a pesar de ser ambos grupos morfológicos identificados en diferentes lugares del mundo, las funcionalidades adscriptas varían de acuerdo al contexto (ver más abajo). En otros trabajos publicamos información respecto de la estructura física, métrica, morfológica y microscópica de las colecciones aquí analizadas y, a partir de ellas, hemos concluido que muchas piezas pueden asimilarse a sistemas de armas (Buc 2007, 2010a, b; Loponte 2008). Presentamos, en cada caso, un resumen de dicha información.

Para llevar a cabo este análisis abordamos cada grupo morfológico de manera independiente y evaluamos las distintas propiedades geométricas y dimensionales que los caracterizan. También desarrollamos una serie experimental que tuvo como objetivo evaluar la utilidad del diseño de estos grupos morfológicos en los diferentes sistemas de armas. Asimismo, generamos patrones de rastros microscópicos actuales y luego los comparamos con los obtenidos en las piezas arqueológicas. En todos estos casos, los análisis fueron realizados a bajos y altos aumentos $(50,100$ y 200x) con lupa binocular y microscopio de luz incidental (o metalográfico) de platina invertida.

\section{Análisis de la muestra}

Descripción de los grupos morfológicos:

Puntas líticas

Los cabezales líticos analizados provienen de los sitios La Bellaca 2, Las Vizcacheras, Garín y Punta Canal. Si bien sus relaciones dimensionales, así como sus propiedades balísticas han sido discutidas apropiadamente en otro lugar (Loponte 2008) es interesante destacar aquí algunos rasgos característicos. Todos los proyectiles comparten un diseño básico. Se trata de puntas de silueta triangular, apedunculadas, de base escotada o ligeramente escotada con aletas esbozadas como puede observarse en la Figura 2.

Las puntas muestran relaciones dimensionales similares pero varían en función de la materia prima. En este sentido, las puntas de calcedonia tienen una longitud media de 30 $\mathrm{mm}$, aunque su coeficiente de variación (CV) igual a $36 \%$, mientras que la variable ancho posee una media de $17 \mathrm{~mm}$ y un CV de tan solo el 7\%. Por otro lado, en las puntas de cuarcita el largo medio de $25 \mathrm{~mm}$ con un CV del $24 \%$ mientras que la variable ancho posee una media de $15 \mathrm{~mm}$ y un CV de $21 \%$. De esta manera, se observa que las puntas de calcedonia tienen bases más estandarizadas, a pesar de que la longitud posee una varianza muy alta. En cambio, las puntas de cuarcita son menores en tamaño, pero sus dimensiones están más estandarizadas evidenciado por la baja varianza de sus magnitudes. La Figura 3 grafica estas relaciones, donde vemos que las puntas de cuarcita se ajustan mejor al modelo que postula el gráfico donde la variable largo determina el ancho de las puntas. En este sentido, el coeficiente de determinación solo para las puntas de cuarcita es de 0,63 . Sin embargo, no se han descartado para este modelo aquellas puntas con evidencia de alteraciones debido a la reactivación.

Otro aspecto notable, es que dentro del conjunto de puntas líticas de calcedonia, la relación dimensional entre largo y espesor es negativa ( $c f$. Loponte 2008: 7.2.3). En este sentido, podemos pensar que se optó por maximizar la capacidad de penetración del proyectil, frente a su durabilidad (Loponte 2008). 

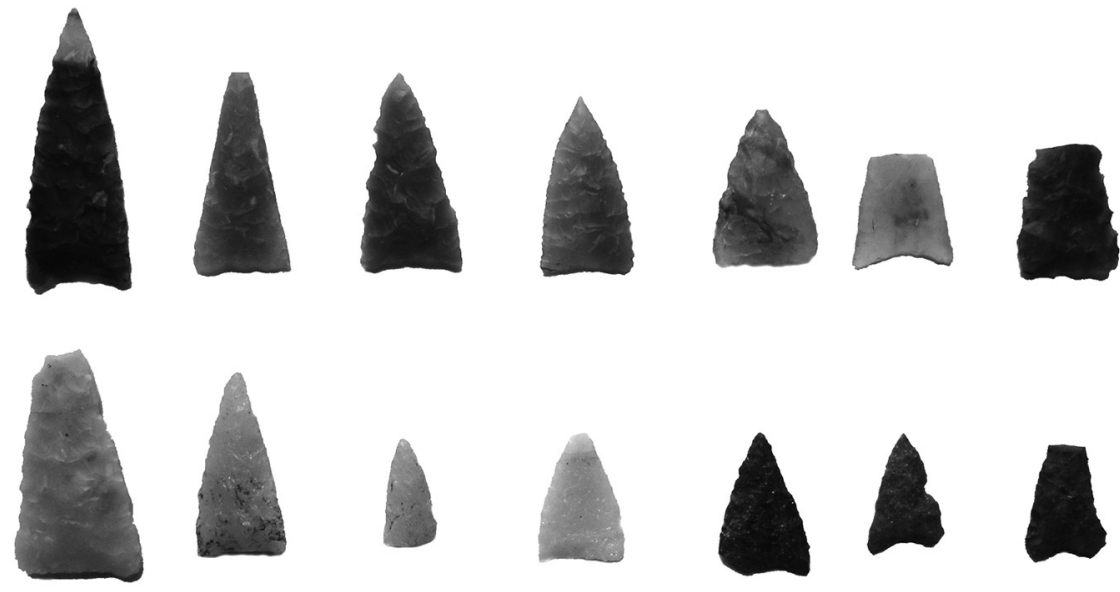

Figura 2. Diseño de cabezales líticos arqueológicos.

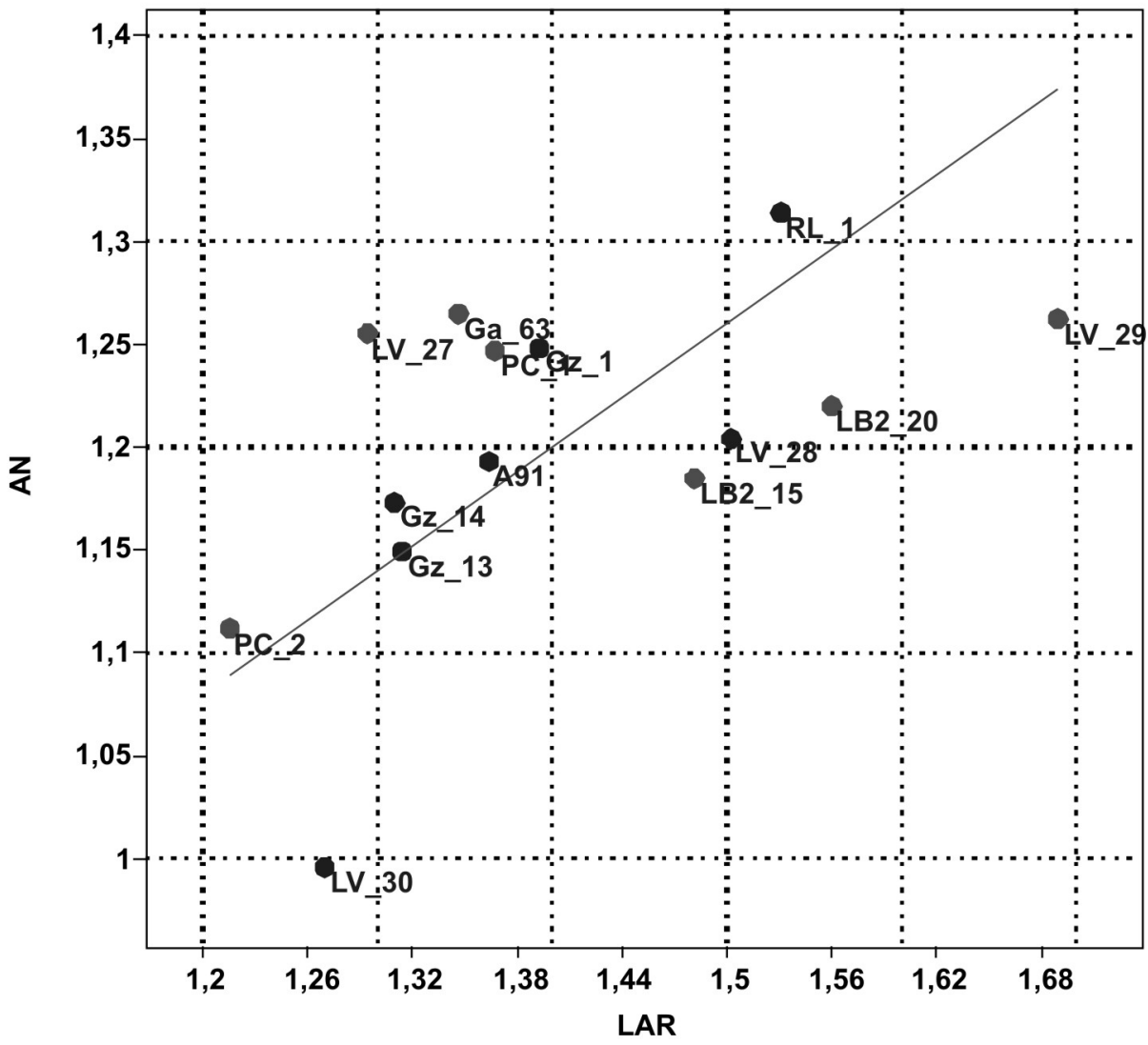

Figura 3. Gráfico de regresión que expresa la relación entre las variables dimensionales "largo" y "ancho" para los cabezales líticos. Los valores del eje Y están expresados en escala logarítmica. Los puntos representan los cabezales líticos de calcedonia, las triangulos los cabezales líticos de cuarcita. 
En cuanto a las materias primas, todas las puntas fueron confeccionadas en rocas alóctonas al HPI. Es importante en este punto, detenernos y analizar ciertos aspectos vinculados con la distribución regional de las materias primas líticas. Si bien el HPI no posee fuentes primarias de rocas con fractura concoidal, existen algunos afloramientos rocosos en regiones aledañas, de calidad variable. Como podemos observar en la Figura 4, la fuente más próxima es la Formación Ituzaingó, que aflora en la margen izquierda del río Paraná y que posee estratos de areniscas cuarcíticas con diferente grado de silicificación. Asimismo, el lecho del río Uruguay provee rodados silíceos secundarios, aptos para la talla. De igual manera, tanto la margen izquierda como derecha del río Uruguay presenta afloramientos primarios de caliza silicificada, conocida como Formación Puerto Yeruá, afloramiento que ha sido caracterizado recientemente (Loponte et al. 2011). Finalmente, existen otras fuentes potenciales de aprovisionamiento lítico, ya más alejadas del ambiente del HPI, como las formaciones rocosas de Sierras Centrales y Tandilia. A esta última corresponde el Grupo Sierras Bayas (GSB) que presenta afloramientos de ftanita y de ortocuarcita. Si bien todas estas fuentes constituyen potenciales sitios de aprovisionamiento, todas las puntas líticas presentes en el HPI fueron confeccionadas con calcedonias y cuarcitas similares a las presentes en el GSB (Loponte 2008; Silvestre 2010). Esto resulta altamente llamativo, ya que la caliza silicificada es una de las materias primas mas explotadas en los sitios del HPI (Silvestre 2011a, 2011b). Asimismo, esta materia prima se encuentra

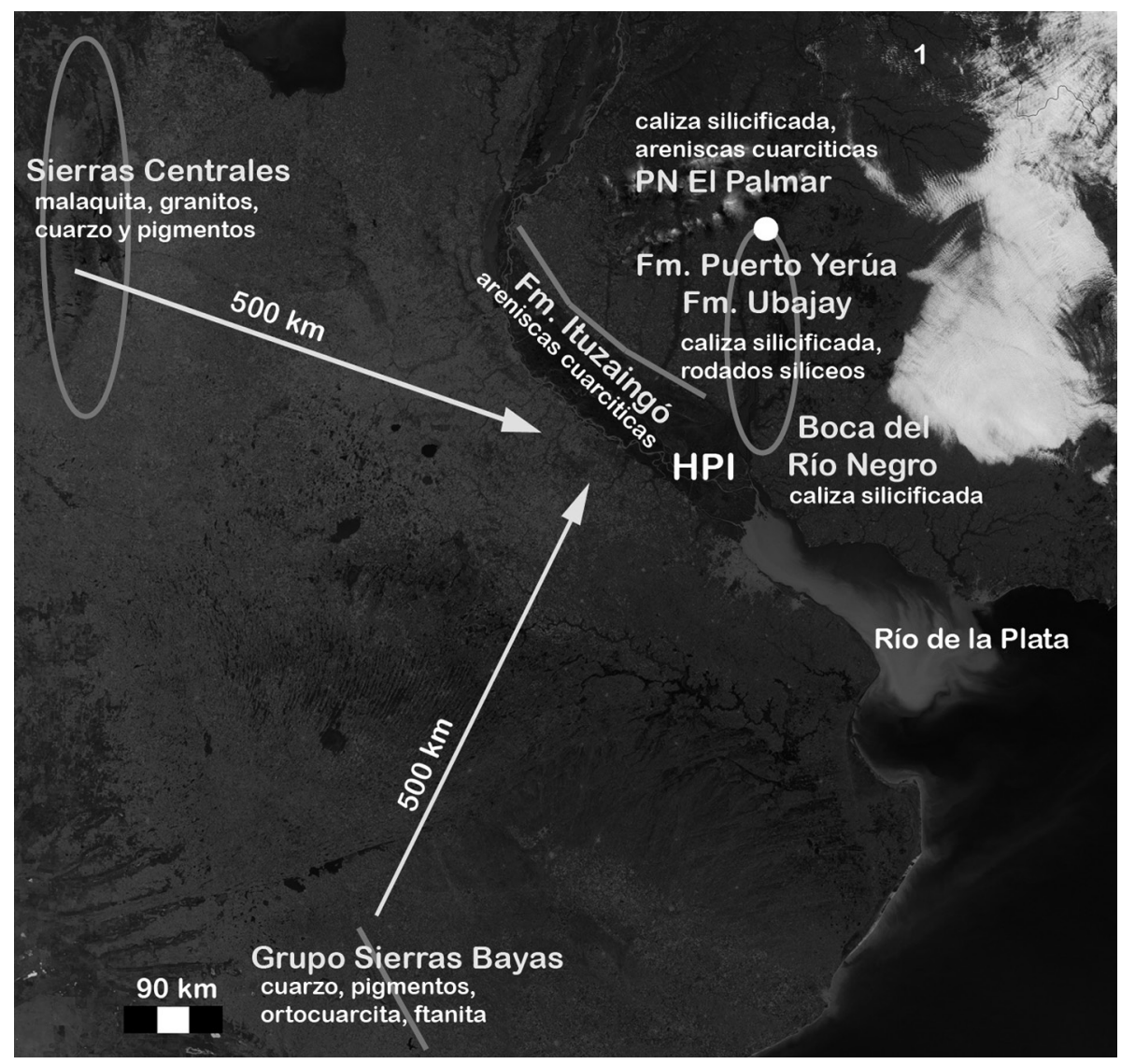

Figura 4. Mapa de distribución regional de materias primas líticas. 
representada en la forma de puntas de proyectil en varios sitios de Uruguay, incluso para la confección de puntas cola de pescado (Bracco et al. 2008; Gascue 2009a, 2009b; Suárez 2009; Vega y Andrade 2004). Esta notoria ausencia de la caliza silicificada como soporte para cabezales líticos podría estar vinculada al alto porcentaje de pérdida de materia prima en la confección de puntas, producto de la gran cantidad de inclusiones de carbonatos que posee la roca y sus consecuentes accidentes de talla reportados en otros trabajos (Gascue 2009a), aunque todavía no ha sido testeado en nuestro caso. Podríamos pensar que se optó por maximizar esta roca para la confección de lascas de filo natural utilizadas para la confección de artefactos óseos, en una economía que se encontraba lejos de las canteras (Buc y Silvestre 2006, 2010; Loponte 2008; Silvestre 2004, 2010).

Si analizamos las frecuencias de las materias primas presentes, las proporciones de puntas confeccionadas en calcedonia superan a las de cuarcita. Esta relación es tenue, pero como dijimos anteriormente, no refleja en absoluto las frecuencias medias de materias primas presentes en los sitios del HPI. Por último, es interesante resaltar que en ninguno de los sitios se han encontrado lascas de adelgazamiento o retoque bifacial (Buc y Silvestre 2006; Loponte 2008).

Puntas óseas pedunculadas (Figura 5a)

Dentro de la gran cantidad de piezas que conforma el conjunto artefactual óseo bajo estudio (aproximadamente 300), se recuperaron sólo dos puntas planas pedunculadas: una fracturada (A 69) y otra entera (LB2 124, Buc 2010b). Estas piezas son de contorno simétrico, planas en ambas caras, tienen aletas angulares y pedúnculo basal (Buc 2010b). De esta manera presentan secciones similares a las puntas de proyectil líticas y poseen, además, un cuidadoso diseño aerodinámico (Buc 2010b; Loponte 2008).

Este tipo de puntas tiene una presencia mayor en sitios del Paraná medio (Buc y Pérez Jimeno 2010; Pérez Jimeno 2007) y otras dos áreas ajenas al Paraná, como son la cuenca del río Salado en Santiago del Estero (Reichlen 1940; Rusconi 1933) y las Sierras Centrales de Córdoba (e.g. Berberian et al. 2011). Sin embargo, en estas dos últimas zonas hay mayor variabilidad morfológica. Hasta el momento, en los Bajíos Ribereños sólo se recuperaron puntas con aletas angulares (Buc 2010b), una de ellas completamente decorada (LB2 124). En otro trabajo (Buc y Pérez Jimeno 2010) sugerimos que esta variabilidad estaría reflejando situaciones estilísticas y que la pieza de La Bellaca 2 podría no estar asociada al sistema de armas propiamente dicho (Buc y Loponte 2007). Luego, discutiendo la muestra de manera más fina, reconocimos la posibilidad de que las puntas con aletas rectas representen estadios de reactivación y que ambas hayan formado parte del sistema de armas (Buc 2010b; Loponte 2008). De hecho, tanto en la pieza de Anahí como en la de La Bellaca 2, a nivel microscópico, se identificó, además de las huellas de manufactura en el cuerpo de la pieza, un patrón de estrías angostas y superficiales, dispuestas de manera paralela entre sí y transversal al eje, en un caso en la aleta y en el otro sobre el pedúnculo (Buc 2010b). Estas son similares a varias series: a) las registradas debajo de los huecos de los arpones (Buc 2007, 2010b) y en las bases de las bipuntas (ver abajo), b) a nuestras propias muestras experimentales resultadas del alisado de vegetales (Buc 2011), y c) las documentadas por Tankersley (1994) como resultado del enmangue en puntas de obsidiana. Por lo tanto creemos que este patrón estaría vinculado al enmangue de las piezas (Buc 2010b). 


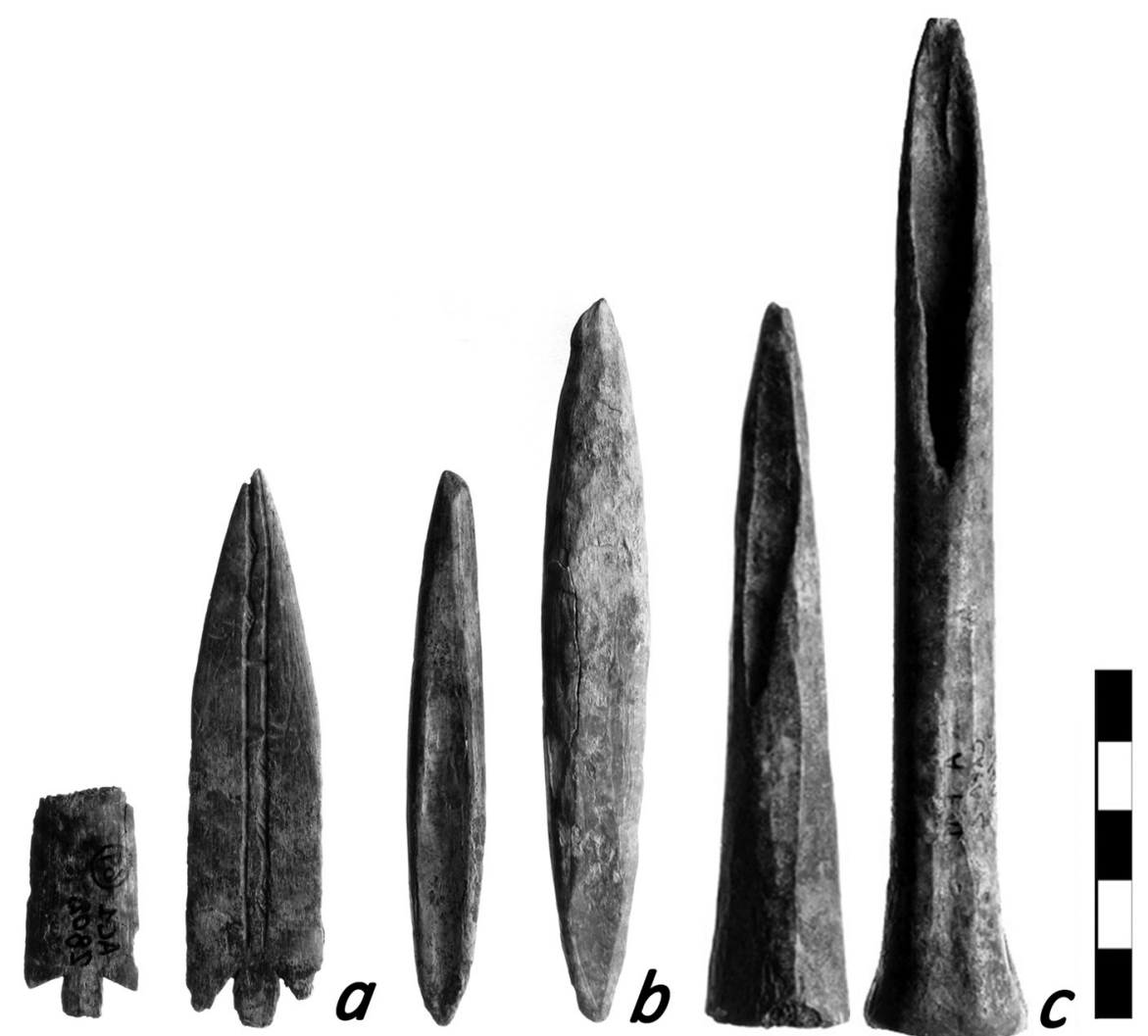

Figura 5a. Puntas óseas discutidas en el trabajo: a) puntas pedunculadas, b) bipuntas, c) puntas ahuecadas.

Los datos métricos del ancho del pedúnculo y el espesor son semejantes en las dos piezas, siendo el primero de $5 \mathrm{~mm}$ y el último de $3 \mathrm{~mm}$. Si consideramos que esta última variable se mantiene constante en muestras provenientes del Paraná medio (con una media total de $2.6 \mathrm{~mm}$ y un desvío estándar de $0.4 \mathrm{~mm}$; Buc y Pérez Jimeno 2010), podemos establecer que existió una tendencia a la estandarización dimensional de las puntas planas pedunculadas en la cuenca del Paraná en variables no afectadas por la reactivación.

\section{Bipuntas (Figura 5b).}

Las bipuntas (Buc 2010a) fueron recuperadas únicamente en La Bellaca 2 con un total de 10 piezas. Este grupo morfológico presenta una distribución mundial tan amplia que las hipótesis funcionales varían de acuerdo a los autores y contextos arqueológicos. Una de ellas, la más general, las adscribe como puntas de proyectil (e.g. Lothrop 1932; Newcomer 1974; Tyzzer 1936). Sin embargo, otros autores las vinculan directamente con la pesca: puntas de arpón (Fontana 1977 [1881]; Lyman 1991; Pokines y Krupa 1997); anzuelos simples (hipótesis tradicional en contextos del Paleolítico superior: Lyman 1991; Rick et al. 2001; Smith 1929 en Tyzzer 1936) y anzuelos compuestos (Lyman 1991). Piezas de morfología similar fueron descriptas como partes del sistema de enmangue de puntas de proyectil (Lahren y Bonnichsen 1974). Para el área de estudio, evaluando el peso, la longitud, la sección y el hueso-soporte 
de las bipuntas, Loponte (2008) sugiere que las mismas serían puntas de proyectil, y que las variaciones métricas al interior de la muestra pueden responder a adecuaciones para distintos sistemas de armas.

En Buc (2010a, 2010b) evaluamos las hipótesis funcionales de las bipuntas mediante el análisis microscópico de la muestra de La Bellaca 2, partiendo de la base de que se esperan diferentes patrones microscópicos en cada caso. Como resultado, encontramos que el ápice, el sector medial y la base de las bipuntas presentan patrones que permiten discriminarlos funcionalmente. En el sector apical del 50\% de las piezas se registra un patrón de estrías cortas, profundas, transversales al eje de la pieza pero paralelas entre sí (Figura 6a). Este patrón es del mismo tipo que el documentado en los cabezales de arpones y las puntas ahuecadas arqueológicas (comparar Figura 6a con 15 e-f; Buc 2010b; Musali y Buc 20011) y ha sido discutido como producto de impacto (Buc 2010a, 2010b). En el sector mesial las piezas sólo presentan la superficie redondeada o huellas de manufactura por lo cual suponemos que éste no fue el sector funcionalmente activo. En la base, el 50\% de la muestra presenta un patrón de estrías finas, agrupadas y transversales que podrían ser de enmangue (Figura 6b y Buc 2010a, 2010b). Claramente, la estructura microscópica de las bipuntas apoya la hipótesis general de cabezales de armas o anzuelos completos, aunque el análisis de las estructuras morfológica, métrica y física, permite una discusión más detallada.

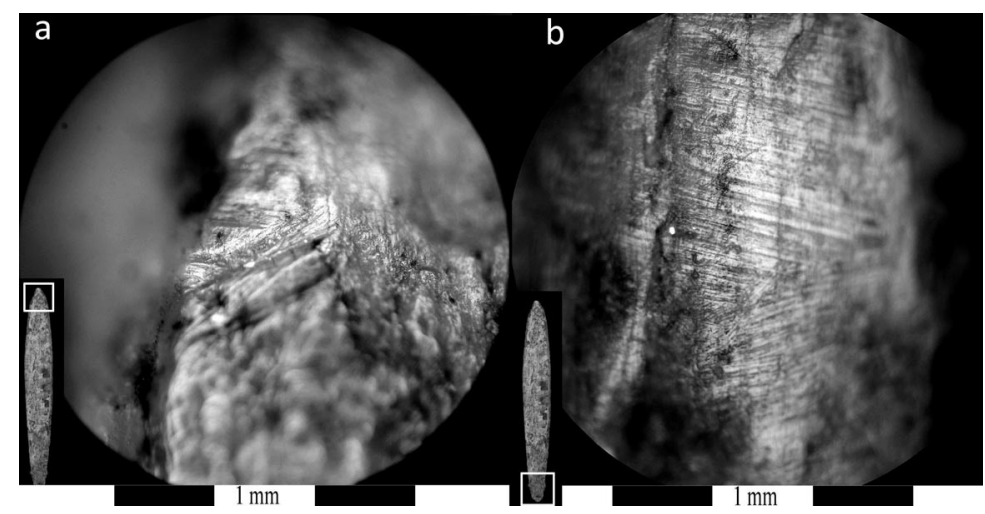

Figura 6. Bipunta arqueológica (LB2 52): a. Estrías transversales cortas y profundas registradas en el extremo apical (200X); b. Estrías finas y agrupadas en el sector basal (200X).

Para que las bipuntas conformen extremidades de un arpón o anzuelo diseñados para la pesca, deben enfrentar la resistencia de la presa (de manera similar a los requerimientos presentados para las puntas de arpón, Buc 2007), por lo que su morfología debería ser la de un diente (e.g. Chiara 1987:128 en Herberts 1998: Figura 40a-b para un ejemplo más cercano; Pokines y Krupa 1997). De modo contrario, el perfil de las bipuntas aquí analizadas no es combado sino recto. Sin embargo, no podemos descartar esta idea sin programas experimentales orientados en este sentido.

La estructura física, morfológica, métrica y de rastros microscópicos del conjunto de las bipuntas de La Bellaca 2 se ajusta con más confianza a la hipótesis de cabezales de flecha o lanza, a partir de varios puntos largamente discutidos en Buc (2010a, 2010b): la resistencia del material óseo, el engrosamiento mesial de las piezas que otorga mayor capacidad de penetración, y la extremidad basal adelgazada que facilita el enmangue. De hecho, la 
morfología es similar a los cabezales de madera utilizados por los pescadores amazónicos actuales (Loponte 2008) y los registrados para los mbayá-guaycurú (Chiara 1987: 127 en Herberts 1998: Figura 39a-b). Métricamente, las bipuntas varían en largo de 100 a $40 \mathrm{~mm}$, valor que se correlaciona con los de ancho y espesor que van de 15 a $7 \mathrm{~mm}$ y de 10 a $3 \mathrm{~mm}$, respectivamente. Es decir que hay piezas más largas, anchas y espesas y otras más cortas, angostas y delgadas. No obstante, en relación al espesor, todas caen dentro de los parámetros presentados por Guthrie (1983) para puntas de proyectil óseas (media de $10 \mathrm{~mm}$ ). Loponte (2008) reconoce, además, una cierta tendencia de las bipuntas mayores a $70 \mathrm{~mm}$ a estar hechas sobre asta, y las menores, sobre hueso ${ }^{1}$. Según este autor las primeras estarían vinculadas a sistemas de puntas que pueden ser arrojadas a mano (como lanzas) o con propulsor, mientras las segundas, entrarían en el rango de las puntas de flecha. Esto es consistente con la estructura métrica, las bipuntas más largas son más pesadas ( $\sim 9$ gr) y tienen anchos de entre 11 y 15 mm, que según el modelo de Ratto (2003) estarían vinculadas a astiles pesados, como las lanzas propulsadas a mano. Las bipuntas más cortas son más livianas ( $\sim 6 \mathrm{gr})$ y tienen anchos menores a $10 \mathrm{~mm}$, acordes a un astil liviano, como podría ser el de una flecha para ser arrojada con arco (cf. Buc 2010b; Ratto 2003). Si, además, consideramos los criterios de Ratto (2003) establecidos para puntas líticas, el perfil binconvexo simétrico, el contorno simétrico y su tamaño mediano permitiría clasificarlas como puntas de aerodinámica perfecta. Si bien no podemos extrapolar este modelo de manera directa al material óseo, la experiencia que presentamos en este trabajo tiene el objetivo de evaluar esta posibilidad, utilizando bipuntas de tamaño relativamente pequeño para ser propulsadas con arco.

\section{Puntas ahuecadas}

Las puntas ahuecadas (Figura 5c) son piezas de contorno simétrico, ápice de sección circular o cóncavo-convexa (según la materia prima utilizada sea asta o hueso, respectivamente) y ahuecadas en la base. En general son concebidas como "cabezales de armas" en un sentido amplio, aunque algunos autores locales se animan a definirlas como "puntas de lanza" (Lothrop 1932: Figura 44) o arpones apedunculados (Caggiano 1977). En un trabajo previo (Buc 2007) evaluamos esta hipótesis a partir del análisis de las 26 puntas ahuecadas disponibles y concluimos que éstas no funcionaron como cabezales de arpón separables. Las diferencias en las estructuras físicas, morfológicas y métricas de ambos grupos morfológicos no permiten definirlos como variaciones en diseño del mismo artefacto (Buc 2007, 2010b).

En cuanto a la estructura física, las puntas ahuecadas están hechas sobre asta o metapodios de cérvidos, en partes prácticamente iguales (Buc 2007, 2010b). Ambos elementos tienen buenas propiedades materiales para realizar actividades de penetración, siendo más rígido el metapodio (es decir que es menos resistente al impacto) y más elástica el asta (tolerando mejor el stress; Guthrie 1983; Scheinsohn 1997; ver también Buc 2007, 2010 b para un análisis detallado). Los análisis microscópicos realizados muestran distintos patrones de rastros que sugieren que las puntas ahuecadas fueron instrumentos versátiles utilizados para diferentes actividades. Algunas están asociadas a actividades de perforación de materiales blandos (tipo "punzón") y otras muestran huellas del mismo tipo que las registradas en los arpones, que podrían ser de impacto. Por ello propusimos que las puntas ahuecadas constituyen un diseño versátil que pueden haber sido utilizados como cabezales de armas (Buc 2007, 2010b). Las variables métricas, finalmente, se distribuyen de manera heterogénea al interior del grupos de las puntas ahuecadas, aunque esto no es suficiente para separar sub-conjuntos (Buc 2010b). El espesor mantiene una media de 10 
$\mathrm{mm}$, lo cual se alinea con los valores óptimos para puntas de proyectil (cf. Guthrie 1983). El hueco basal tiene un diámetro medio de $10 \mathrm{~mm}$ que, de acuerdo a los valores presentados por Ratto (2003) se asocia a astiles relativamente pesados. En este caso, entonces, la penetración estaría dada por la masa, por lo que podemos sugerir que las puntas ahuecadas sirvieron como puntas de lanza y no de flecha, como sería el caso anterior, donde es crítica la trayectoria de vuelo. De acuerdo a estas ideas, la experiencia presentada en este trabajo apunta a evaluar la efectividad del diseño de puntas ahuecadas del HPI como punta de lanza. Si bien estas pueden corresponder a la utilización de sistemas de propulsores, en este caso la utilizamos como arma fija.

\section{Aproximación experimental}

Si bien se han desarrollado muchos trabajos experimentales con puntas de proyectil, la mayoría se realizaron utilizando puntas líticas (e.g. Ahler 1971; Aoyama 2005; Browne 1940; Dockall 1997; Frison 1989; Martínez y Aschero 2003; Odell y Cowan 1986; Shea 1988, 1990); siendo muy pocos los de puntas óseas (Arndt y Newcomer 1986; Frison y Zeimens 1980; Guthrie 1983; Pokines 1998; Pétillon 2006; Rozoy 1992; Tyzzer 1936). Más escasas aún son las investigaciones en donde se presentan comparaciones entre ambos tipos de materiales (Guthrie 1983). Gracias a estos trabajos y a otros publicados desde la etnoarqueología (Hiscock 1994; Greaves 1997; Griffin 1997; Shott 1989), sabemos que diferentes sistemas de armas (arco y flecha; lanza; propulsores) emplean tipos de puntas particulares y que cada materia prima tienen distinta resistencia mecánica (Ellis 1997; Knecht 1997).

Como mencionamos, el propósito general de esta experiencia fue ensayar la efectividad diferencial de las puntas líticas y óseas como parte de los distintos sistemas de armas. Asimismo, mantuvimos tres ejes específicos: 1) evaluar la performance de la materia prima lítica; 2) probar la utilidad de las bipuntas y de las puntas ahuecadas como puntas de flecha y lanza fija, respectivamente y 3) generar un patrón de rasgos microscópicos para analizar los distintos grupos morfológicos en el registro arqueológico. Especialmente en el caso de las puntas óseas, como mencionamos, el registro inicial de las distintas piezas arqueológicas (puntas ahuecadas, bipuntas y arpones) ha brindado rastros muy particulares en el ápice que podrían ser producto de impacto. Por ello, y sabiendo de la importancia de la forma y materia prima de los artefactos en la génesis de los microrrastros (LeMoine 1991), independientemente de mantener constante las acciones (de impacto y penetración en tejido blando) fue necesario realizar la experiencia con los diferentes diseños de puntas.

En primer lugar buscamos replicar puntas de morfología y materia prima similar a las arqueológicas (Figura 7 y 8). Para los cabezales líticos se utilizó ftanita proveniente del Grupo Sierras Bayas, materia prima ampliamente utilizada tanto en el HPI como en toda la región pampeana. Si bien dentro del registro arqueológico de los sitios bajo estudio, existen puntas en otras materias primas, como la ortocuarcita, se analizaron únicamente proyectiles confeccionados en ftanita, material con el cual estamos familiarizados con el proceso de formación de microrrastros y su evaluación microscópica ${ }^{2}$. Para las puntas óseas utilizamos hueso de Ovis aries (oveja) y asta de Cervus elaphus (ciervo colorado) en estado seco. Nuestro objetivo no fue replicar las técnicas de manufactura de las piezas, pero, dado que necesitamos tener un registro microscópico de las superficies "en estado 0 ", las observamos antes de su utilización con diferentes dispositivos ópticos. 


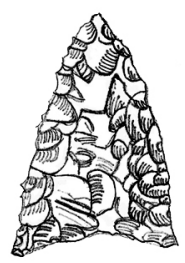

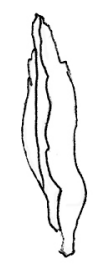

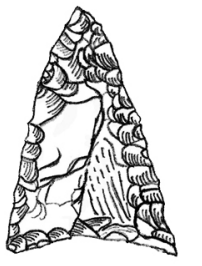

Ex-2
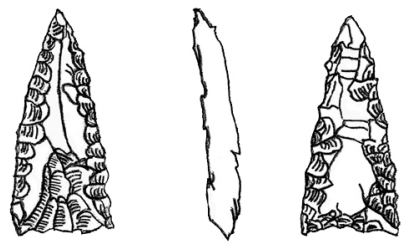

Ex-3
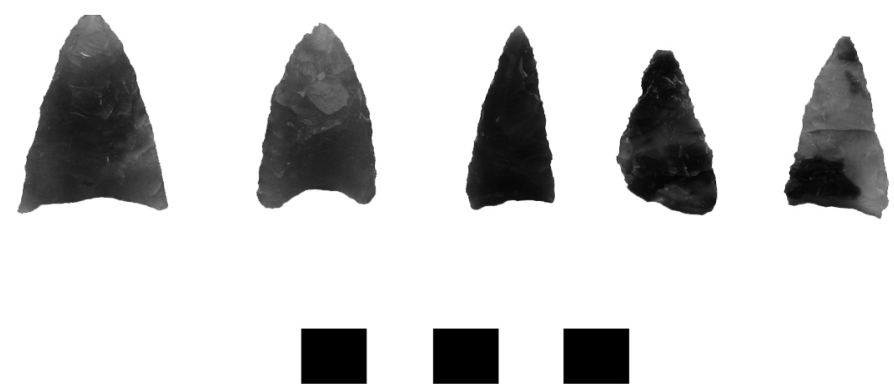

Figura 7. Réplicas de cabezales líticos utilizados en la experimentación.

Hubo dos instancias de experimentación. En la primera sólo utilizamos proyectiles óseos reproduciendo los dos grupos morfológicos aquí discutidos. Por un lado, utilizamos piezas de morfología similar a las bipuntas arqueológicas, siendo del grupo de las más pequeñas con aproximadamente $50 \mathrm{~mm}$ de largo (Figura 8a). Los cabezales fueron insertados en un astil de madera (pino) y sujetados con cinta de teflón. En esta ocasión los astiles no fueron emplumados y tenían un largo total de $320 \mathrm{~mm}$ aproximadamente. Colocamos una parrilla costal de $\mathrm{O}$. aries a $8 \mathrm{~m}$ de distancia del tirador, elevada a $45^{\circ}$ del suelo natural. La experiencia la realizó un arquero experimentado utilizando un arco recurvo de 62 pulgadas y 40 libras. Al colocar el objetivo de esta manera buscamos que las puntas fallidas no golpeen contra superficies que puedan alterarlas de manera significativa, pero no evitamos la pérdida de una punta. En los proyectiles restantes $(n=3)$ logramos entre uno y cinco impactos por cada uno (Tabla 1). En dicha oportunidad ensayamos también con las puntas ahuecadas, de mayores dimensiones (aproximadamente $100 \mathrm{~mm}$, Figura 8b), una hecha sobre asta de C. elaphus y la otra sobre un metapodio de $O$. aries. Utilizamos las dos puntas insertadas en un astil para impactar sobre la misma parrilla costal pero esta vez de manera directa reproduciendo el funcionamiento de las lanzas. Las piezas resistieron los repetidos impactos, no sólo contra tejido blando sino que procuramos asestar el hueso intencionalmente. Con una de las piezas (E07-4) logramos 50 impactos pero la otra (E07-7) quedó alojada en el hueso al impacto 34 (Tabla 1). A pesar de esto, ninguna de las puntas sufrió fracturas macroscópicas. 


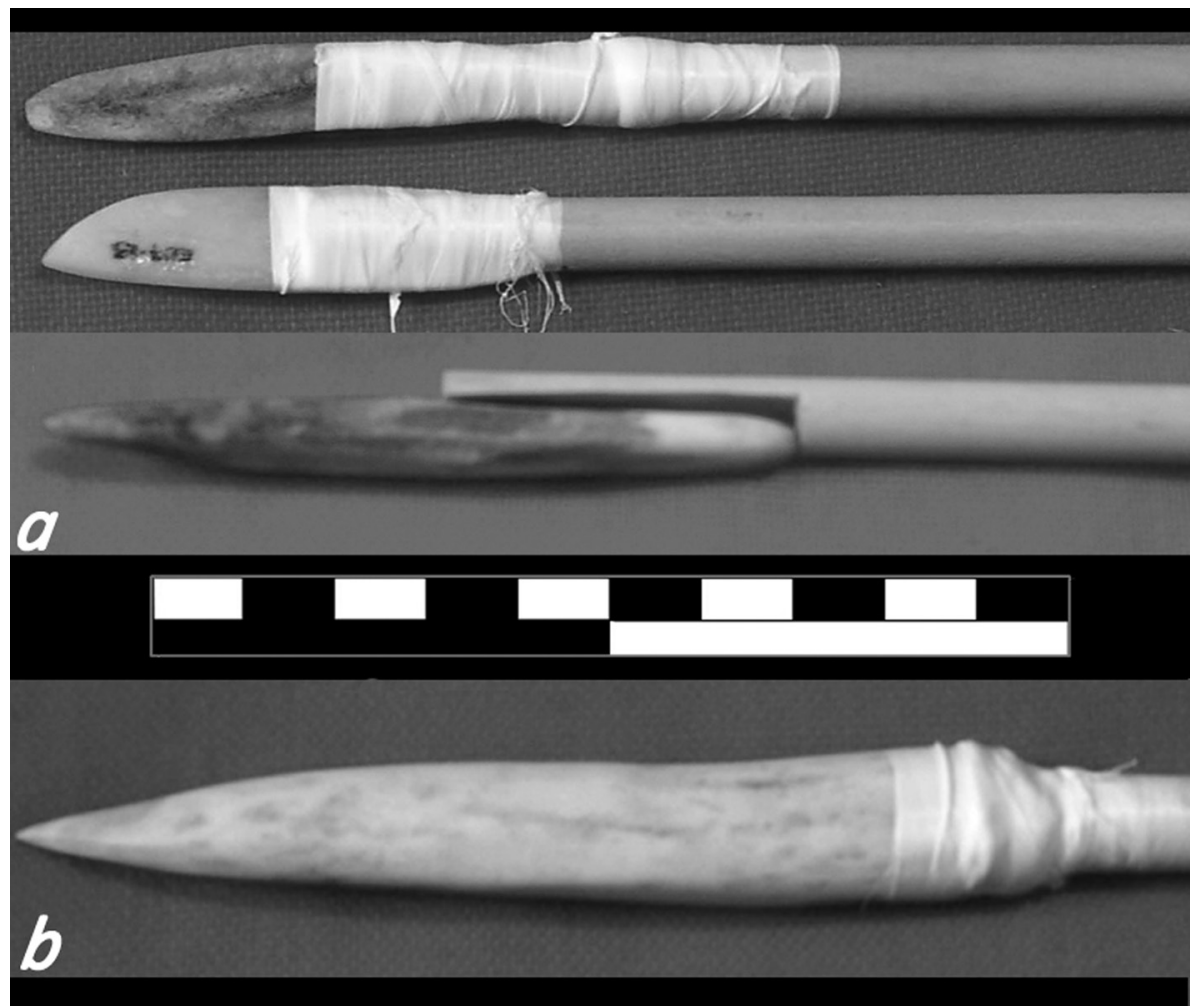

Figura 8. Réplicas de cabezales óseos utilizados en la experimentación: a) bipuntas, b) punta ahuecada.

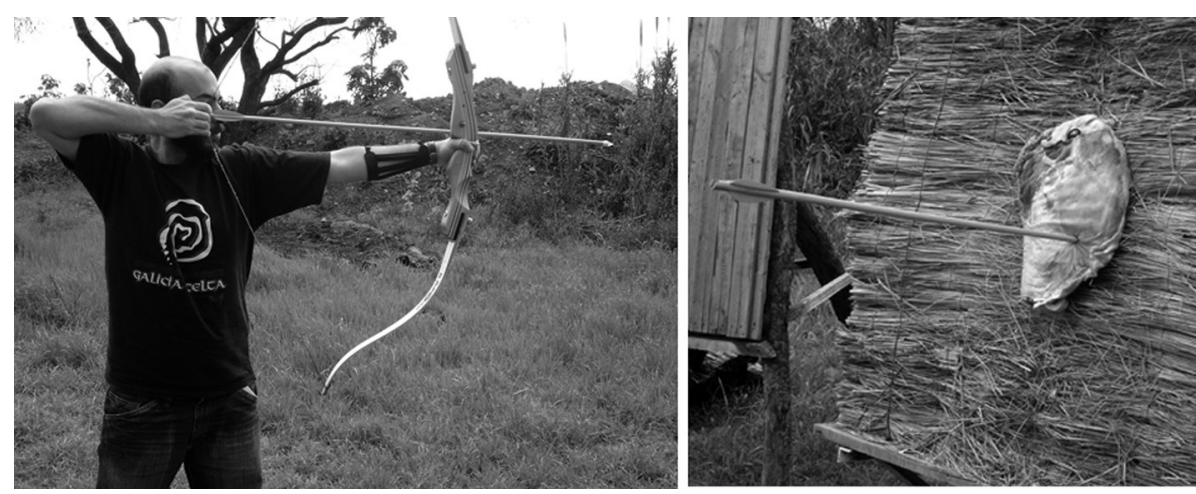

Figura 9. Experimentación de puntas proyectadas con arco arrojadas sobre parrilla costal de Ovis aries colocada sobre fardo de paja.

Para la segunda experiencia reutilizamos una de las bipuntas de hueso e incorporamos las líticas. En este caso los astiles fueron emplumados y la parrilla costal de O. aries fue colocada sobre un fardo de paja en el Club Universitario de Arquería de Buenos Aires (Figura 9). Si bien este sistema no impidió que se extraviaran proyectiles, disminuyó la probabilidad. Como resultado de la experiencia, la bipunta ósea recibió 21 impactos y permaneció intacta. 


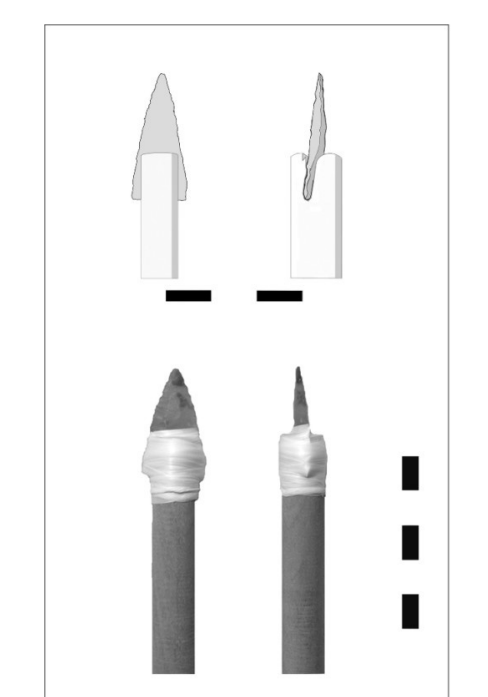

Figura 10. Réplicas de cabezales líticos

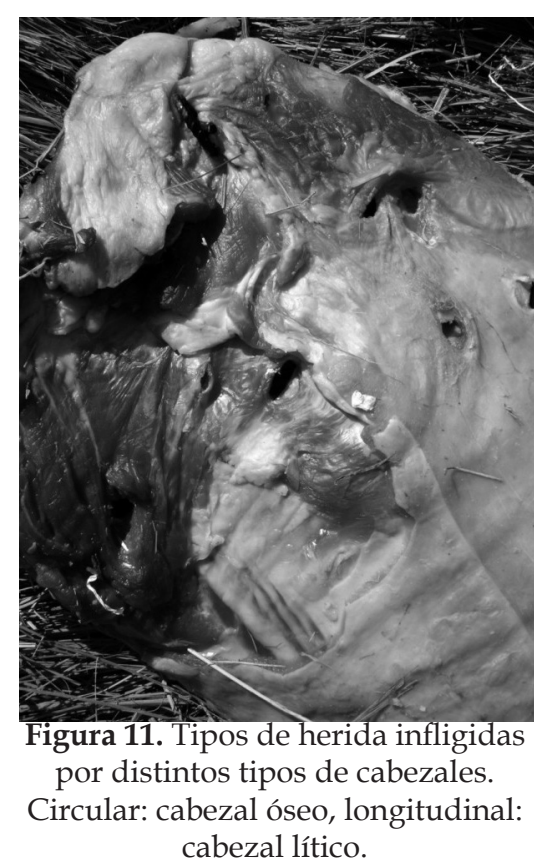

En el caso de las puntas líticas, el arquero se colocó en un intervalo entre 5 y $12 \mathrm{~m}$ y las flechas tenían un largo total (punta y astil) de $112 \mathrm{~cm}$. Se conocen por lo menos tres tipos básicos de procedimientos de enmangue (Keeley 1982, con algunas variaciones, ver Moss 1987; Rots 2002), pero aquí los cabezales fueron insertados en ranuras realizadas en los astiles y envueltos en teflón, como en el caso de las bipuntas óseas (Figura 10). Como se desprende de la Tabla 2 luego de la experiencia, todas las puntas líticas presentaron daños a ojo desnudo, dos resultaron fracturadas sin poder continuar con su utilización y una se perdió en la simulación.

Una vez terminada la experiencia se realizaron registros fotográficos del tipo de daño infligido en el tejido blando de la carcasa de acuerdo al uso de cabezales de diferentes materias primas. De manera consistente con el diseño y material de las puntas, encontramos que mientras las líticas produjeron daños longitudinales, las óseas generaron heridas de contorno circular sobre el tejido blando (Figura 11). La misma relación fue documentada por Pétillon y Letorneaux (2008). Los autores consideraron directamente los daños ocasionados sobre los elementos óseos a partir de una serie experimental propia de puntas óseas, retomando los resultados de Smith et al. (2007) de puntas líticas.

\section{Análisis microscópico}

\section{Puntas líticas}

Los resultados de los análisis microscópicos de las puntas líticas pueden agruparse en tres grandes categorías de datos. En primer lugar, se registraron macrofracturas (Aoyama 2005; Bergman y Newcomer 1983; Dockall 1997; Fischer et al. 1984; Odell y Cowan 1986; Shea 1988, 1990, 1993) en todas las puntas experimentales. Fracturas longitudinales se presentan en dos ejemplares, aunque su tamaño no impidió que pudieran seguir utilizándose (Figura 


\begin{tabular}{cc|c|c|c|c|c|} 
& H-soporte & Acción & Material & Estado & Lapso & Grupo morfo. \\
\hline E07-11 & Asta & PERDIDA & PERDIDA & PERDIDA & PERDIDA & bipunta \\
\hline E07-12 & Asta & Impacto & hueso y carne & húmedo & $1 \mathrm{imp}$ & bipunta \\
\hline E07-13 & Mtp & Impacto & hueso y carne & húmedo & $21 \mathrm{imp}$ & bipunta \\
\hline E07-14 & Mtp & Impacto & hueso y carne & húmedo & $5 \mathrm{imp}$ & bipunta \\
\hline E07-15 & Mtp & Fricción & Hilos sisal & húmedo & $15 \mathrm{~min}$ & bipunta \\
\hline E07-4 & Asta & Impacto & hueso y carne & húmedo & $50 \mathrm{imp}$ & p. ahuecada \\
\hline E07-7 & Mtp & Impacto & hueso y carne & húmedo & $34 \mathrm{imp}$ & p. ahuecada \\
\hline
\end{tabular}

Tabla 1. Síntesis experiencia con bipuntas óseas como puntas de flecha.

\begin{tabular}{c|c|c|c|c|c|c}
$\mathrm{N}^{0}$ punta & Tierra & Paja & Blanco & Total de tiros & \%Penetración & Estado* \\
\hline 6 & 1 & 9 & 5 & 15 & 30 & Fracturada \\
\hline 4 & 0 & 3 & 9 & 12 & 75 & Perdida \\
\hline 1 & 0 & 8 & 7 & 15 & 46,66 & Lascada \\
\hline 2 & 0 & 4 & 29 & 33 & 87,87 & Lascada \\
\hline 7 & 0 & 1 & 8 & 9 & 88,88 & Lascada - fracturada \\
\hline
\end{tabular}

Tabla 2. Experimentación con puntas líticas. Datos de propulsión. * Estado de la punta luego de la experimentación.

12a). Fracturas distales y transversales (Dockall 1997) se encontraron en dos ejemplares, lo cual tuvo como resultado que las puntas no pudieran ser reutilizadas. Un solo ejemplar presenta una fractura lateral (Figura 12c).Además, todas las piezas presentan diferentes tipos y frecuencias de microlascados en sus filos (Figura 12e). Todos estos rastros se producen por fallas mecánicas de la materia prima frente a situaciones de alto impacto (Dockall 1997; Odell y Cowan 1986).

En segundo lugar, si bien los microrastros han sido propuestos por varios autores como un gran sesgo dentro de los análisis tradicionales de proyectiles líticos (Dockall 1997; Shea 1988, 1990), en nuestro caso no son concluyentes. No se desarrollaron micropulidos, exceptuando por dos puntas que presentan zonas abradidas en sectores localizados de sus filos, muy poco desarrollados, con sectores brillantes e irregulares en su extensión. Pensamos que estas abrasiones pueden ser resultado en alguna medida, del contacto de las puntas con el soporte del blanco (ver arriba). En cambio, si se registraron estrías en tres de los cuatro ejemplares examinados. Se trata de estrías cortas, anchas y profundas, paralelas al eje del artefacto, tanto en los filos como en los extremos distales, aunque escasas en frecuencia. Por último, se registraron rastros de enmangue. Brevemente podemos decir que la génesis de estos rastros se vincula tanto con acciones de impacto, como con el movimiento de la pieza dentro del astil (Jensen 1994; Keeley 1982; Moss 1987; Rots 2002, 2003, 2004). Se registraron bright spots (parches de pulidos aislados, bien desarrollados; Rots 2002) y pulidos tanto en las aletas como en las bases (Figura 13a y c). La característica principal es que no tienen direccionalidad, siguen los rasgos de la microtopografía de la roca, y poseen un límite claro. Las estrías se encuentran presentes en todos los casos examinados, en la base, en ocasiones paralelas a la base y en otras perpendiculares al eje del artefacto. Por último, son abundantes los microlascados localizados tanto en aletas como bases, con forma de media luna o escalonadas, producto del impacto de la punta contra el astil.

\section{Bipuntas}

Obtuvimos registros microscópicos de cada una de las piezas experimentales, sin embargo, ni en entre las primeras, donde el máximo fue de cinco impactos, ni en la segunda, en la cual 

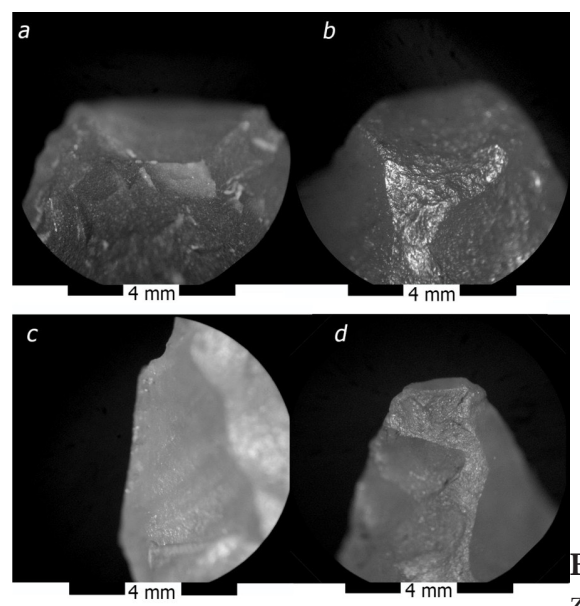

Figura 12. Rastros macroscópicos detectados en cabe-

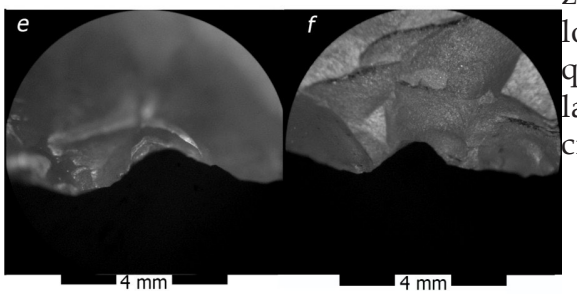
zales líticos experimentales y arqueológicos. a: fractura longitudinal experimental, b: fractura longitudinal arqueológica, c: fractura lateral experimental, d: fractura ateral arqueológica, e: microlascado experimental, f: microlascado arqueológico.

Figura 13. Microrrastros reconocidos en cabezales líticos experimentales y arqueológicos. a: bright spots experimentales, b: bright spot arqueológico, c: bright spots experimentales, $\mathrm{d}$ : redondeamiento de la arista de la base del cabezal con estrías perpendiculares al eje de la arista, en cabezal arqueológico, e y f: residuos detectados en la zona de enmangue de cabezales arqueológicos probablemente producto del uso de un intermediario óseo.

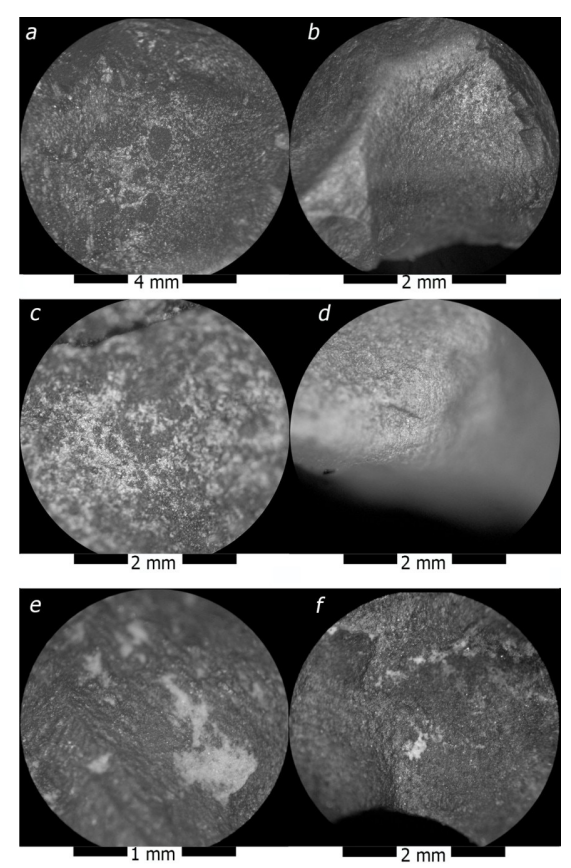

logramos un total de 21 impactos, observamos cambios de la microtopografía del cabezal que no sea el redondeado (Buc 2010b, 2011; Figura 14). Lamentablemente, el redondeado es la huella que diferentes autores señalan como resultado del impacto (Arndt y Newcomer 1986; Pétillon 2006; Pokines 1998; Tyzzer 1936) pero es débil como rasgo comparativo para el análisis de instrumentos óseos arqueológicos ya que puede ser producido igualmente por otros factores. 


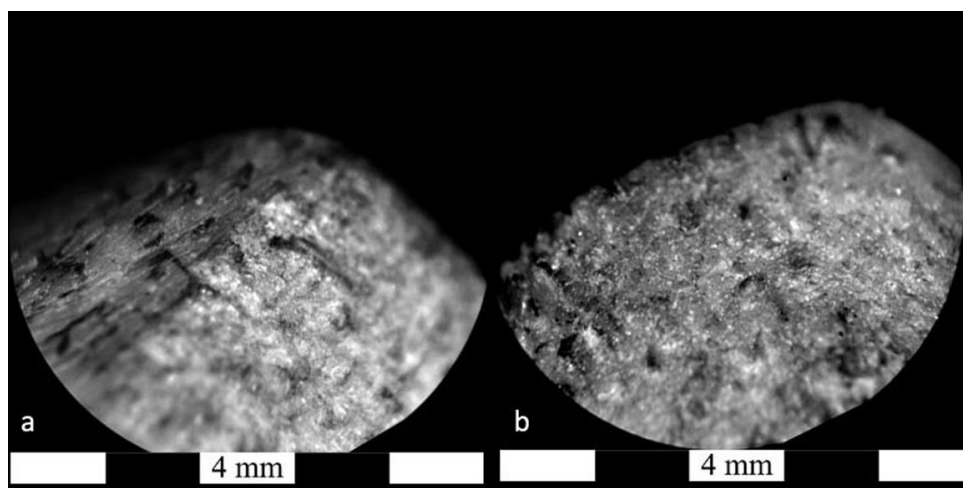

Figura 14. Bipunta experimental (E07-12), extremo apical: a: antes de la utilización; b: luego de la utilización (50X).

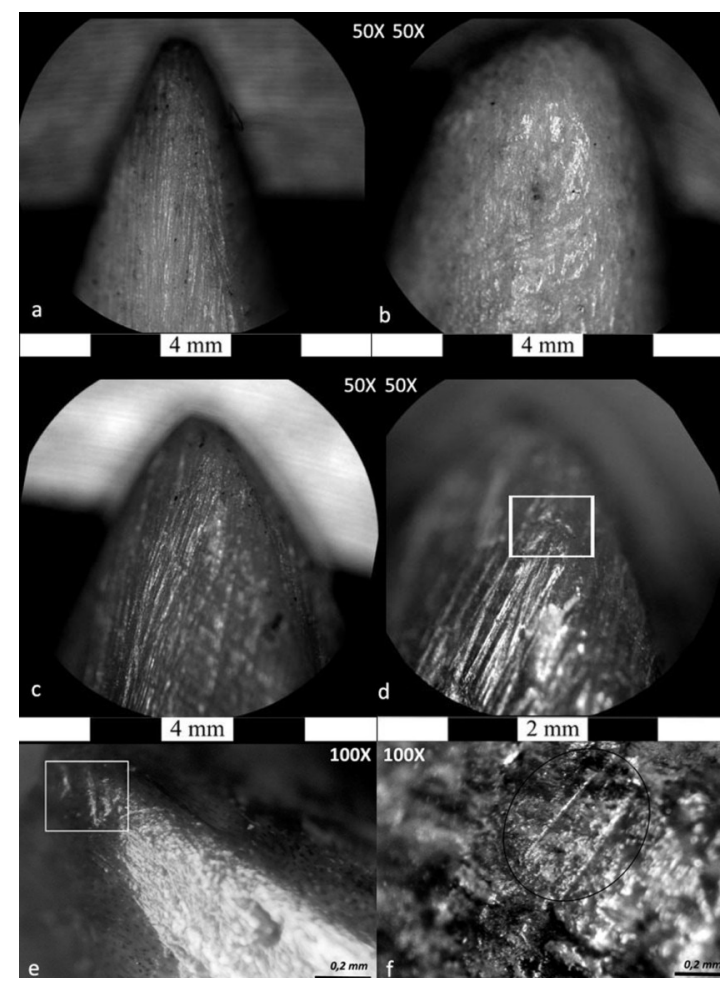

Figura 15. a: E07-04 antes de la utilización (50X); b: E07-04 luego de la utilización, detalle redondeado de la superficie (50X); c: E07-07 antes de la utilización (50X); d: E07-07 luego de la utilización, detalle estría transversal corta sobre el extremo apical (100X); e: punta ahuecada arqueológica (A44; 100X); f: punta ahuecada arqueológica (A36; 100X)

\section{Resultados}

Puntas ahuecadas

En las puntas ahuecadas los rastros fueron diferentes en una y otra pieza. En E07-4, tras 50 impactos, notamos la superficie redondeada de manera intensa, aunque preservando las huellas de manufactura y sin otro rasgo asociado (Figura $15 \mathrm{a}-\mathrm{b}$ ). E07-7, que es la pieza retenida en el hueso y con menos cantidad de impactos, muestra un redondeado menos marcado. Pero, en cambio, en el extremo apical registramos una estría corta y profunda 
localizada de manera transversal al eje de la pieza (Figura 15 c-d). Posiblemente esto sea producto del impacto y perforación del hueso ya que éste fue el único rastro significativo registrado en toda la muestra experimental (Buc 2010b, 2011b). Este tipo de estría es sugestivo ya que es similar a las registradas en puntas ahuecadas, bipuntas y arpones arqueológicos (ver arriba Figura 6a, Figura 5 comparar d con e y f).

\section{Puntas líticas}

Si bien todas las puntas arqueológicas presentan evidencias de alteraciones post depositacionales, en todas se registraron rastros, excepto en un caso (Ga 1) donde no se pudo reconocer ningún otro rasgo característico, mas allá de presentar fractura longitudinal (Tabla 2). Los tipos de fractura mas representados son la longitudinal y lateral (Figura 12b y d). En ningún caso se registraron fracturas distales o transversales. Los microlascados son abundantes en todas las muestras, sobre todo en los filos en zonas adyacentes a los extremos distales (Figura 12f). Igual que en los casos experimentales, no se registraron micropulidos, aunque si estrías cercanas a las puntas, paralelas al eje del artefacto, aunque de morfología variable. En cuanto a los rastros de enmangamiento, estos se encuentran presentes en todas las piezas, aunque son de diferente naturaleza. Bright spots y pulidos, se encuentran en todos los casos, excepto en dos, tanto en la base de las puntas como en las aletas (Figura 13b y d). Microlascados en forma de media luna de boca profunda, fueron registrados en tres casos (Figura 12f). Se encuentran localizados en los laterales de la punta en zonas medias proximales. Estos rastros se vincularon experimentalmente con el proceso de enmangue (Rots 2002, 2003; Shea 1988, 1993). Estrías cortas, anchas y profundas se encontraron en la mayoría de los artefactos.

Por último, se registraron otros tipos de rastros no reconocidos experimentalmente (Figura 13 e y f). Se trata de residuos, que pensamos podrían ser de origen animal (hueso) pero frente a los cuales preferimos ser cautos. Análisis EDAX (espectroscopia de rayos por energía dispersiva) podrán determinar en el futuro la naturaleza de estos rastros. Preliminarmente, podemos decir que estos rastros podrían ser vinculados con el uso de un intermediario orgánico que funcione de manera similar al detallado por Lahren y Bonischen (1974) para las puntas Clovis (ver también Lyman et al. 1998).

\section{Puntas planas pedunculadas}

Si bien el espesor de estas piezas resulta muy delgado de acuerdo a los $10 \mathrm{~mm}$ presentados por Guthrie (1983) como el óptimo para puntas de proyectil óseas, no podemos concluir que las puntas planas no afronten los requerimientos mecánicos de las puntas de proyectil ya que hemos visto que presentan rastros que podrían ser de uso. Como señaló Loponte (2008), dicha particularidad habría sido superada por el diseño que resalta la agudeza de las aristas lo que permite que las puntas actúen por corte, de manera más similar a un cabezal lítico, y no por impacto, particularidad que le atribuye la plasticidad ósea y para lo cual sí se requiere el espesor señalado por Guthrie (ver también Buc 2010b). Además, el ancho del pedúnculo de $5 \mathrm{~mm}$ concuerda, dentro del modelo de Ratto, con la estructura métrica de las puntas de flecha asociada a astiles livianos (Ratto 2003). En este punto, dadas las condiciones particulares de las situaciones de caza (e.g. Ellis 1997) y considerando que estas puntas son cabezales de armas arrojadizas, no descartamos que la mencionada decoración de la pieza tenga como finalidad señalar la identidad de un determinado cazador o artesano. Sin embargo, resta una experimentación controlada sobre este grupo morfológico que 
permita constatar, además de un patrón de microrastros de impacto y enmangue, la posibilidad aerodinámica de las piezas, tal como realizamos en los restantes grupos morfo-funcionales (GM-F).

\section{Bipuntas}

Ya presentamos los resultados del análisis microscópico en las piezas arqueológicas por lo que aquí discutiremos el caso experimental. A pesar de no haber obtenido un registro controlado de huellas de impacto sobre las bipuntas, como resultado de nuestra experiencia pudimos constatar que dicho diseño es efectivo si se emplea como punta de proyectil. Tal como discute Knecht, el engrosamiento del sector medio aumenta la capacidad de penetración de la punta en la presa y facilita la apertura de la herida (Knecht 1997), requisitos necesarios para las puntas óseas cuya eficacia, a diferencia de las líticas, no radica en el corte, como vimos en los daños registrados en el blanco (ver también Pétillon y Letorneaux 2008). Nuestros resultados prácticos refuerzan, entonces, la hipótesis de que las bipuntas óseas hayan sido puntas de proyectil tal como sugería el análisis de las estructuras métricas, físicas, morfológicas y microscópicas discutidas anteriormente.

Sin embargo se necesitan futuros trabajos experimentales para probar la eficiencia de las posibles bipuntas como puntas de lanza. Más allá de la discusión puntual sobre la adscripción de las bipuntas al sistema de arco y flecha o de lanza, bien pudieron ser cabezales intercambiables: dada la alta disposición de materia prima y los bajos costos de manufactura, las bipuntas habrían sido una solución para ambos sistemas (Loponte 2008). La gran diferencia entre uno y otro se encuentra en la menor precisión de las lanzas con respecto al arco y flecha ( $c f$. Churchill 1993; Loponte 2008). De tal manera, es posible que en esos casos se minimicen los riesgos de fractura del cabezal utilizando asta por sobre el hueso que es un material más flexible ( $c f$. Guthrie 1983) y, en consecuencia, más adecuado para cumplir una actividad de penetración mediada por el impacto (Scheinsohn 1997). El hueso, de mayor rigidez sería menos adecuado para sistemas mecánicamente exigentes como el de lanzas que para el de arco y flecha. Sin embargo, al ocurrir todas las bipuntas en el sitio La Bellaca 2 tampoco descartamos que esta variabilidad esté respondiendo a situaciones particulares de este sitio (Buc 2010b). Asimismo, dada la amplia dispersión de este GM-F en otros lugares del mundo, entendemos que su diseño generalizado puede haber cumplido diferentes funciones según el contexto ya que con pequeñas variaciones morfológicas puede adecuarse a diferentes rangos métricos. En pocas palabras, con costos energéticos muy bajos, este GM-F satisface altos requerimientos mecánicos, incluyendo aquellos que otros autores proponen para las bipuntas, como anzuelos o intermediarios de puntas de proyectil.

\section{Puntas ahuecadas}

Del mismo modo que el caso anterior, los resultados microscópicos de estas piezas fueron presentados, y nos focalizamos aquí en el aspecto experimental. Si bien no podemos ser concluyentes en cuanto a la identificación microscópica tampoco en este caso, el único registro de impacto obtenido de la serie experimental es una huella similar a las documentadas en ciertas puntas ahuecadas arqueológicas que, a su vez, son del mismo tipo que las bipuntas y arpones arqueológicos (Buc 2007, 2010b, 
2011b). A nivel teórico, siguiendo el trabajo de Arndt y Newcomer (1986) podemos suponer que estas huellas (cortas, transversales al eje y profundas) sean producto de la compresión del material óseo ante el impacto con una superficie más dura, sin embargo, es necesario considerar que estamos ante sólo una pieza experimental y hecha sobre un material particular (metapodio de O. aries) por lo que no podemos definir esta huella como característica del impacto de proyectiles (Buc 2010b, 2011b).

Nuestra experiencia, en definitiva, confirma la efectividad de las puntas ahuecadas como puntas de lanza de mano tal como sugerimos a partir de las estructuras morfológicas, físicas y métricas discutidas. Sin embargo, no descartamos que, dado la variabilidad de los patrones microscópicos (ver arriba), las puntas hayan utilizadas alternativamente como perforadores. Si bien el hueco basal, rasgo que define al GM-F, es crítico en la hipótesis de cabezales de armas y no es relevante a la utilización de las piezas como perforadores, tampoco lo impide. No descartamos que sean piezas de fácil reciclado o que tengan diferente funcionalidad por sitio (Buc 2010b).

\section{Discusión y conclusiones}

A partir de nuestra experiencia pudimos constatar que tanto el hueso, como la piedra y el asta requieren un diseño particular, resultado de adecuar sus propiedades mecánicas a la actividad destinada (en este caso, de punta de proyectil; cf. Guthrie 1983; Knecht 1997; Scheinsohn 1997). Si bien este no es el espacio para profundizar, es interesante retomar la vieja discusión sobre cabezales y tipos de armas.

Los esfuerzos etnoarqueológicos pierden consistencia al intentar relacionar ambas partes de manera inequívoca ya sea porque los grupos utilizan el mismo cabezal para distintas armas o diferentes proyectiles para la misma función, dependiendo de la situación particular de encuentro de las presas (Ellis 1997; Hiscock y Bleed 1997). Por ello se ha discutido largamente sobre cuáles son las variables críticas para adscribir puntas a sistemas de armas, considerando principalmente el peso (Fenenga 1953; Rozoy 1992; Hughes 1998) y el ancho del hombro (Thomas 1978; Shott 1997). También se incluyen las relaciones dimensionales y la forma aerodinámica de las piezas, como por ejemplo, el trabajo de Ratto (2003). Tomando estas variables, en nuestro caso, podemos sugerir el empleo de arcos para los cabezales líticos, las puntas planas pedunculadas y las bipuntas de tamaño pequeño. Las puntas ahuecadas y los cabezales de arpón, si bien no pueden excluirse como partes de un sistema de propulsión, muestran propiedades que sugieren que fueron empleados como cabezales de armas impulsados directamente por el brazo humano (Loponte 2008). Resta por experimentar con las bipuntas de gran tamaño como cabezales de lanzas o dardos.

El modelo presentado nos permite considerar un manejo eficiente de las propiedades del material lítico y óseo por los grupos cazadores-recolectores del HPI (Buc 2010b; Buc y Silvestre 2006; Silvestre 2010). La gran variedad de puntas presentadas es acorde no sólo a la existencia de distintos sistemas de armas, sino también a la disponibilidad diferencial y propiedades particulares de cada materia prima. Dada la escasez de rocas, podríamos pensar a las puntas líticas como artefactos multifuncionales que cumplieron la función de puntas de proyectil como parte de un amplio repertorio de actividades. Sin embargo, los datos funcionales no apoyan esta hipótesis: los microrastros registrados en las puntas arqueológicas se asocian únicamente al impacto (i.e. no existen micropulidos diagnósticos que 
den cuenta del uso de las puntas en otras actividades como por ejemplo corte). Seguramente, los cazadores-recolectores del HPI adecuaron la flexibilidad y resistencia diferencial de cada material a los distintos tipos de armas, en función de los riesgos de pérdida que implican las distintas presas y situaciones de caza.

Finalmente, debe señalarse que es necesario continuar realizando análisis microscópicos específicos, ya que existen importantes diferencias entre los materiales líticos y los óseos. El hueso, al ser un material anisotrópico (Ellis 1997; Knecht 1997; Odell y Cowan 1986) es relativamente plástico y por ello, luego del impacto solo se registra el redondeado de las superficies. En cambio, la naturaleza quebradiza de la materia prima lítica sometida a fuerzas de alto impacto genera modificaciones no sólo micro sino también macroscópicas (Dockall 1997; Ellis 1997; Odell y Cowan 1986; Shea 1988). Si bien seguimos conformado un registro de microrrastros comparativos, nos queda por evaluar las posibilidades de piezas no consideradas en este trabajo como las lascas de filo natural con formas punzantes o la gran cantidad de puntas óseas fragmentadas. Dentro de la colección de sitios relevada aquí se recuperaron, por ejemplo, puntas de sección plano-convexa o cóncavo-convexa de base recta con todo el canal medular expuesto. Este diseño es similar a las provenientes de sitios del Paraná medio (Pérez Jimeno 2007: fig.7.7-7.9; Pérez Jimeno y Buc 2009) y a las que se ilustra etnográficamente como cabezales para los mbayá-guaicurú hechas en tacuara (Herbert 1998: Figura 39c). En Buc 2010b señalamos que de acuerdo al modelo de Ratto (2003), por su estructura morfológica estas puntas tienen una aerodinámica imperfecta (sección plano o cóncavo convexa, contorno simétrico y tamaño muy grande). Sin embargo, resultan livianas para cabezal de lanza de mano, por lo que no descartamos que sean piezas recicladas o bien parte del sistema de propulsores.

Discutir la combinación de todas estas posibilidades permite ir completando el cuadro tecnológico del HPI durante el Holoceno tardío. Entendemos que los grupos de cazadoresrecolectores no disponían de un toolkit con una conformación "una presa, un sistema, una punta". Si bien existieron sistemas relativamente especializados (como los arpones; Musali y Buc 2011) el manejo de la tecnología ofreció una gran variabilidad de armas que permitieron ampliar el abanico de nichos y especies explotadas de manera significativa ( $c f$. Loponte 2008). Así, las puntas de flechas líticas y óseas funcionaron de manera integrada respondiendo, más allá del conocimiento y manejo de la materia prima, a las contingencias de la situación caza. En este sentido, no descartamos la posibilidad de envenenamiento de los cabezales como sugieren algunas crónicas históricas (Azara 1943[1847]; Dobrizhoffer 1967 [1783-1784]), ni la posible utilización de algunas lascas de filo natural como cabezales líticos (cf. Ellis 1997; Hiscock y Bleed 1997; Odell 1988; Odell y Cowan 1986). A su vez, este sistema de captura estaría combinado con el de lanzas fijas y arrojadas con propulsor, elaboradas a partir de puntas óseas y seguramente también de madera. En el caso del HPI la versatilidad de este sistema alcanza a especies terrestres como a los recursos acuáticos e incluso a las eventualidades bélicas.

\section{Agradecimientos}

Agradecemos a Donald Jackson y a Leonardo Paulides por la confección de las puntas líticas experimentales y a Marcelo Morales por realizar la experiencia de arquería. El departamento de materiales de CIDETEF (especialmente Alejandro Reynoso) permitió el acceso a los microscopios utilizados. Kazuo Aoyama, Alice Choyke, Steven Churchill, Jean Marc Pétillon, Sandra Olsen y Veerle Rots aportaron bibliografía e importantes comentarios. La evaluación del artículo, asimismo, colaboró con la comprensión del mismo. 


\section{Notas}

${ }^{1}$ Arbitrariamente, para dividir las bipuntas en largas o cortas, se considera como umbral los $70 \mathrm{~mm}$.

2Para un análisis de los programas experimentales desarrollados en ortocuarcita y sus procesos de formación de microrastros ver Castro 1994 y Leipus 1999.

\section{Bibliografía citada}

Acosta, A.

2005. Zooarqueología de cazadores-recolectores del extremo nororiental de la provincia de Buenos Aires (humedal del río Paraná inferior, Región pampeana, Argentina). Tesis Doctoral, Universidad Nacional de La Plata.

Ahler, S. A.

1971 Projectile Point Form and Function at Rodgers Sheltev, Missouri. Missouri Archaeological Society Research Series 8. Columbia, MO: Missouri Archaeological Society.

Ames, K. M.

2005. Intensification of food production on the Northwest Coast and elsewhere. The Northwest Coast, Foragers or Farmers? (ed. por D. Duer y N. Turner), pp 64-94. University of Washington Press, Seattle.

Aoyama, K.

2005. Classic Maya Warfare and Weapon. Spear, Dart, and Arrow Points of Aguateca and Copan. Ancient Mesoamerica 16: 291-304.

Arndt, S. y M. Newcomer

1986. Breakage Patterns on Prehistoric Bone Points: An Experimental Study. Studies in the Upper Paleolithic of Britain and Northwest Europe (ed. por D. A. Roe), BAR 296: 165-173, Oxford.

Arrizurieta, M.P., N. Buc, B. Mazza, L. Mucciolo, J. Musali, F. Parisi, D. Pau, M. Pérez, M. Poggi, R. Silvestre.

2010. Nuevos aportes a la arqueología del sector continental del humedal del Paraná inferior. Arqueología Argentina en el Bicentenario de la Revolución de Mayo, J.R. Bárcena y H. Chiavazza (Eds.), Mendoza.CD ROM: Tomo V: 1793-1798.

Azara, Félix de

1943 [1847]. Descripción e historia del Paraguay y del Río de la Plata. Edición digital basada en la edición de Buenos Aires. Editorial Bajel, Buenos Aires.

Bathata, T.

1893. Viaje de un maturrango. Peuser, Buenos Aires.

Berberián, E. ; B. Bixio ; M. M. Bonofiglio; M.C. González Navarro; M.E. Medina; S. Pastor; M.A. Recalde ; D.E. Rivero ; J. Salazar.

2011. Los Pueblos Indígenas de Córdoba. Ediciones del Copista, Biblioteca de Historia. Córdoba. Bergman, C. A. y M. H. Newcomer

1983. Flint Arrowhead breakage: Examples from Ksar Akil, Lebanon. Journal of Field Archaeology 10: 238-243.

Binford, L.

2001. Constructing Frames of Reference. University of California press, Berkeley. 
Bó, R. F. y A. I. Malvárez

1999. El pulso de inundación y la biodiversidad en humedales. Un análisis sobre el efecto de eventos extremos sobre la fauna silvestre asociada a estos sistemas. Tópicos sobre humedales subtropicales y templados de Sudamérica (ed. por A. Malvárez), Buenos Aires, Universidad de Buenos Aires, pp. 147-168.

Bonfils, C.

1962. Los suelos del Delta del Río del Paraná. Factores generadores, clasificación y uso, Revista de Investigación Agraria, INTA VI (3).

Bousman, C. B.

1993. Hunter-gatherer adaptations, economic risk and tool design. Lithic Technology.18 (1/2): 59-86.

Bracco, R., L. del Puerto y H. Inda

2008. Prehistoria y arqueología de la Cuenca de Laguna Merin. Entre la Tierra y el Agua: Arqueología de Humedales de Sudamérica (comp. por D. Loponte y A. Acosta). AINA, Buenos Aires.

Browne, J.

1938. Antiquity of the Bow. American Antiquity 3 (4): 358-359

1940. Projectile Points. American Antiquity 5 (3): 209-213.

Buc, $\mathrm{N}$.

2007. Ser o no ser: arpones y “arpones B" en el humedal del Paraná inferior. Arqueología en las Pampas. (ed. por C. Bayón, A. Pupio, M. I. González, N. Flegenheimer, N. y M. Frère. Tomo I: 325-342 Sociedad Argentina de Antropología, Buenos Aires.

2010a. Bone Bi-points: Testing Functional Hypothesis. En A. Legrand-Pineau, I. Sidéra, N. Buc, E. David y V. Scheinsohn, Ancient and Modern Bone Artefacts from America to Russia. Cultural, technological and functional signature. BAR International Series 2136: 217-225.

2010b. Tecnología ósea de cazadores-recolectores del humedal del Paraná inferior. En D. Loponte y A. Acosta (Comp.), Series Monográficas, Arqueología de la Cuenca del Plata. Buenos Aires : Instituto Nacional de Antropología y Pensamiento Latinoamericano.

2011a. Algo más que simples puntas. Explorando la variabilidad en la tecnología ósea del Paraná inferior. En M. R. Feuillet Terzaghi; Ma. Belen Colasurdo: J. Sartori y S. Escudero (Eds.) Avances y perspectivas en la arqueología del Nordeste. Santísima Trinidad, Buenos Aires. pp. 59-68.

2011b. Experimental Series and Use-Wear in Bone Tools. Journal of Archaeological Science 38: 546-557.

Buc, N. y D. Loponte

2007. Bone tool types and microwear patterns: Some examples from the Pampa region, South America. Bones as Tools: Current Methods and Interpretations in Worked Bone Studies, (ed. por C. Gates St-Pierre, y R. B. Walker). BAR International Series 1622: 143-157, Oxford .

Buc N. y L. Pérez Jimeno

2010. Puntas para la comparación. Tecnología ósea en el Paraná Inferior y Medio. En M. A. Gutiérrez, M. De Nigris, P. M. Fernández, M. Giardina, A. F. Gil, A. Gil, A. Izeta, G. Neme y H. D. Yacobaccio (eds.), Zooarqueología a principios del siglo XXI: aportes teóricos, metodológicos y casos de estudio. Ediciones del Espinillo (Buenos Aires), p. 439-451. 
Buc, N. y R. Silvestre

2006. Funcionalidad y complementariedad de los conjuntos líticos y óseos en el humedal del nordeste de la Pcia. de Buenos Aires: Anahí, un caso de estudio. Intersecciones en Antropología 7:129-146.

2010. Distribución de artefactos líticos y óseos en el humedal del Paraná inferior. Arqueología de Cazadores-Recolectores en la Cuenca del Plata (ed. por G. Cocco y R. Feulliet Terzaghi). Centro de Estudios Hispanoamericanos, Santa Fe.

Burkart, R., N. Bárbaro, R. Sánchez y D. Gómez

2000. Ecoregiones de la Argentina. Programa de Desarrollo Institucional Ambiental. Administración de Parques Nacionales, Buenos Aires.

Caggiano, M. A.

1977. La práctica de la pesca por arponeo en el Delta del Paraná. Relaciones de la Sociedad Argentina de Antropología XI: 101-106.

Castro, A.

1994. Estudios de análisis funcional de material lítico: un modelo alternativo de clasificación tipológica. Tesis doctoral. La Plata, Facultad de Ciencias Naturales y Museo, UNLP.

Cavallotto, J. L., R. Violante y G. Parker

2004. Sea-level fluctuations during the last 8600 year in the de la Plata River (Argentina). Quaternary International 114: 155-165.

Churchill, S.

1993. Weapon Technology, Prey Size Selection, and Hunting Methods in Modern HunterGatherers, Implications for Hunting in the Palaeolithic and Mesolithic. Archaeological Papers of the American Anthropological Association 4 (1): 11-24.

Dobrizzhofer, M.

1967 [1783-1784]. Historia de los Abipones. Universidad Nacional del Nordeste, Resistencia.

Dockall, J. E.

1997. Wear Traces and Projectile Impact: A Review of the Experimental and Archaeological Evidence. Journal of Field Archaeology 24 (3): 321-331.

Ellis, C.

1997. Factors influencing the use of stone projectile tips. Proyectil Technology (ed. por H. Knetcht), pp. 37-74. Plenum Press, New York.

Fenenga, F.

1953. The weights of chipped stone points: a clue to their functions. Southwestern Journal of Anthropology 9: 309-323.

Fernández de Oviedo y Valdés, G. 1944 [1526]. Historia General y Natural de las Indias. Islas y Tierra-Firme del Mar Océano. Libro XXIII, cap. XII. Editorial Guarania, Asunción del Paraguay.

Fischer, A., P. V. Hansen, y P. Rasmussen 1984. Macro and Micro Wear Traces on Lithic Projectile Points. Journal of Danish Archaeology 3: 1946.

Fontana, L. J.

1977 [1881]. El Gran Chaco. Solar Hachette, Buenos Aires. 
Frison, G. C.

1989. Experimental Use of Clovis Weaponry and Tools on African Elephants. American Antiquity 54 (4): 766-784.

Frison, G. y G. Zeimens

1980. Bone Projectile Points: An Addition to the Folsom Cultural Complex. American Antiquity 45 (2): 231-237.

Gascue, A.

2009a. Tecnología lítica y patrones de asentamiento en la cuenca del Arroyo Grande (Soriano) Arqueología Prehistórica Uruguaya en el Siglo XXI (comp. por J.M. López Mazz y A. Gascue), pp. 133-150. Biblioteca Nacional - Facultad de Humanidades y Ciencias de la Educación, Montevideo.

2009b La tecnología lítica desarrollada por los habitantes prehistóricos del Arroyo del Perdido (Soriano-Uruguay). Arqueología Prehistórica Uruguaya en el Siglo XXI (comp. por J.M. López Mazz y A. Gascue), pp. 117-130. Biblioteca Nacional - Facultad de Humanidades y Ciencias de la Educación, Montevideo.

Greaves, R. D.

1997. Hunting and multifunctional use of bows and arrows. Projectile Technology (ed. por H. Knecht), pp. 287-320. Plenum Press, New York.

Griffin, P. B.

1997. Technology and Variation in arrow design. Projectile Technology (ed. por H. Knecht), pp. 267-286. Plenum Press, New York.

Guthrie, D.

1983. Osseous Projectile Point: Biological Considerations Affecting Raw Material Selection and Design Among Paleolithic and Paleoindian Peoples, Animals and Archaeology: 1. Hunters and their Prey (Ed por J. Clutton-Brock y C. Grigson), 163: 274-294. BAR International Series, Oxford.

Herberts, A.L.

1998. Os Mbayá-Guaicurú:área, assentamento, subsistência e cultura material. Tesis de Maestría, Universidad do Vale do Rio dos Sinos-UNISIONS. MS.

Hiscock, P.

1994. The end of points. Archaeology in the North: Proceedings of the 1993 Australian Archaeological Association Conference (Ed. por M. Sullivan, S. Brockwell y A. Webb.) North Australia Research Unit, Australian National University, Canberra.

Hiscock, R. y P. Bleed

1997. Each According to Need and Fashion. Projectile Technology (ed. por H. Knecht), pp. 345-368. Plenum Press, New York.

Hughes, S.

1998. Getting to the point: Evolutionary change in prehistoric weaponry. Journal of Archaeological Method and Theory 5 (4): 345-408. 
Jensen, J. H.

1994. Flint tools and Plantworking. Hidden traces of Stone Age technology. A use wear study of some Danish Mesolithic and TRB implements. Aarhus University Press, Aarhus.

Keeley, L.

1982. Hafting and retooling: effects on the archaeological record. American Antiquity 47 (4): 798-809.

Kelly, R. L.

1995. The Foraging Spectrum. Diversity of Hunter-Gatherer Lifeways. Smithsonian Institution Press, Washington DC.

Kidder, A. V.

1938 Arrow-Heads or Dart Points, American Antiquity 4 (2): 156-157.

Knecht, $\mathrm{H}$.

1997. Proyectil Points of Bone, Antler and Stone. Experimental Exploration of manufacture and use. Projectil Technology (ed. por H. Knetcht), pp. 191-213. Plenum Press, New York.

Lahren, L. y R. Bonnischen

1974. Bone Foreshafts from a Clovis Burial in Southwestern Montana. Science 11 Vol 186 (4159): $147-150$

Leipus, M. E. P.

1999. Análisis funcional: Caracterización de los microrastros de uso en materias primas líticas de la Región Pampeana. Actas del XII Congreso Nacional de Arqueología Argentina. I: 345-354. Universidad Nacional de La Plata, La Plata.

LeMoine, G.

1991. Experimental Analysis of the Manufacture and Use of Bone and Antler Tools among the Mackenzie Inuit. Ph. D. Dissertation, Alberta, Canada, University of Calgary.

Loponte, D.

2008. Arqueología del Humedal del Paraná inferior (Bajíos Ribereños Meridionales), Instituto Nacional de Antropología y Pensamiento Latinoamericano, Secretaría de Cultura de la Nación, Arqueología de la Cuenca del Plata, Vol.1, Buenos Aires.

Loponte, D., A. Acosta y J. Musali

2006. Complexity among hunter-gatherers from the Pampean region, South America. Beyond Affluent Foragers: Rethinking Hunter-Gatherer Complexity (ed. por C. Grier, J. Kim y J. Uchiyama), pp. 106-125. Oxbow Books, Oxford.

Loponte, D; P. Tchilinguirián y R. Silvestre

2011 Caracterización de afloramientos de calizas silicificadas de la provincia de Entre Ríos (Argentina) y su vinculación con los circuitos de abastecimiento prehispánico. Avances y Perspectivas en la Arqueología del Nordeste (ed. por M. R. Feuillet Terzaghi, M. B. Colasurdo, J. I. Sartori y S. Escudero), pp. 125-139. Santa Fe, Argentina.

Lothrop, S.

1932. Indians of the Paraná Delta River. New York, Annals of the New York Academy of Sciences XXXIII, pp. 77-232. 
Lyman, L.

1991. Archaeology of Umpqua/Eden. Prehistory of the Oregon coast (the effects of inquiry). Academic Press.

Lyman, R. L., M. O'Brien y V. Hayes

1998. A Mechanical and Functional Study of Bone Rods from the Richey-Roberts Clovis Cache, Washington, U.S.A. Journal of Archaeological Science 25: 887-906

Martinez, J. G. y C. A. Aschero

2003. Proyectiles experimentales: Inca Cueva 7 como caso de estudio. Cuadernos FHyCSUNJu 20:351-364.

Moss, E.

1987. Polish $G$ and the question of hafting. Le Main et l'Outil: Manches et Enmanchements Préhistoriques. Trabaux de la Maison de l'Orient 15: 97-101.

Musali, J.

2010. El rol de la pesca entre los grupos humanos de la baja cuenca del Plata (Ictioarqueología de conjuntos prehispánicos del Holoceno tardío en el humedal del río Paraná inferior). Tesis Doctoral. Facultad de Filosofía y Letras, Universidad de Buenos Aires. Ms

Musali, J. y N. Buc

2009. El uso de armas vinculadas a la pesca entre los aborígenes que habitaron el humedal del Río Paraná inferior. Una aproximación experimental. Armas prehispánicas: múltiples enfoques para su estudio en Sudamérica. (ed. por J. Martínez y D. Bozutto). En prensa.

Newcomer, M.

1974. Study and Replication of Bone Tools from Ksar Akil (Lebanon). World Archaeology 6: 138-153.

Odell, G.

1988. Addressing Prehistoric Hunting Practices through Stone Tool Analysis. American Anthropologist 90: 335-356.

Odell, G. y F. Cowan

1986. Experiments with Spears and Arrows on Animal Targets. Journal of Field Archaeology 13 (2): 195-212.

Orians, G. H. y N. E. Pearson

1979. On the theory of central place foraging. Analysis of Ecological Systems (ed. por D. J. Horn, R. D. Mitchell y G. R. Stairs), pp. 154-177. The Ohio State University Press, Columbus.

Paucke, F.

1944[1829] Hacia allá y para acá (una estada entre los indios Mocovíes 1749-1767). Instituto Cultural Argentino-germano-Universidad Nacional de Tucumán, Tucumán.

Pérez Jimeno, L.

2007. Investigaciones arqueológicas en el sector septentrional de la llanura aluvial del Paraná -margen santafesina-: La variabilidad del registro arqueológico. Tesis Doctoral. Facultad de Ciencias Naturales y Museo, Universidad Nacional de La Plata. MS.

Pérez Jimeno L. y N. Buc

2009. Tecnología ósea en la cuenca del Paraná. Integrando los conjuntos arqueológicos del tramo medio e inferior. Mamül Mapu: pasado y presente desde la arqueología pampeana (Ed. por M. Berón, L. Luna, M. Bonomo, C. Montalvo, C. Aranda y M. Carrera Aizpitarte). En prensa, Santa Rosa. 
Pétillon, J. M.

2006. Des Magdaleniens en armes. Technologie des armatures de projectile en bois de cervide du Magdalenien Superieur de la Grotte d'Isturitz (Pyrenees-Atlantiques). Artefacts 10. Editions du CEDARC.

Pokines, J.

1998. Experimental Replication and Use of Cantabrian Lower Magdalenian Antler Projectile Points. Journal of Archaeological Science (25) : 875-886.

Pokines, J. y M. Krupa

1997. Self-barbed antler spearpoints and evidence of fishing. Projectile Technology (ed. por H. Knecht), pp. 240-262. Plenum Press, New York.

Ratto, N.

2003. Estrategias de Caza y Propiedades de Registro Arqueológico en la Puna de Chaschuil (Dpto. de Tinogasta, Catamarca, Argentina). Tesis Doctoral. Buenos Aires, FFyL, UBA.

Reichlen, $\mathrm{H}$.

1940. Recherches archéologiques dans la Province de Santiago del Estero (Rép. Argentine). Journal de la Société des Américanistes 32 (1): 133-237.

Rusconi, C.

1933. Instrumentos óseos trabajados por indígenas prehispánicos de Santiago del Estero.

Revista de la Sociedad de Amigos de la Arqueología VII: 229-250.

Rick T. C., J. M. Erlandson y R. L. Vellanoveth

2001. Paleocostal Marine Fishing on the Pacific Coast of the Americas: Perspectives from Daisy Cave, California. American Antiquity 6 (4): 595-613.

Rots, V.

2002 Bright spots and the question of hafting. Anthropologica et Praehistorica 113: 61-71.

2003 Towards and understanding of hafting: the macro- and microscopic evidence. Antiquity 77: 805-815.

2004 Prehensile wear on flint tools. Lithic Technology 29: 7-32.

Rozoy, J-C.

1992 Expérimentation de lancer de sagaies avec le propulseur. Bulletin des Chercheurs de la Wallonie Tome XXXII : 169-184

Sánchez Labrador, J.

1910 El Paraguay Católico. Imprenta de Coni Hermanos, Buenos Aires.

Scheinsohn, V.

1997 Explotación de materias primas óseas en la Isla grande de Tierra del Fuego. Tesis Doctoral. Buenos Aires, Facultad de Filosofía y Letras, UBA.

Schmidel, U.

1881[1567] Historia y Descubrimiento del Río de la Plata y Paraguay y Brasil. Imprenta y Librería de Mayo, Buenos Aires.

Shea, J. J.

1988 Spear points from the Middle Paleolithic of the Levant. Journal of Field Archaeology 15: 441-450.

1990 A Further Note on Mousterian Spear Points. Journal of Field Archaeology 17: 111- 115. 
1993 Lithic use wear evidence for hunting in the Levantine Middle Paleolithic. Traces et Fonction: Les Gestes Retrouvés (ed. por P. Anderson, S. Beyries, M. Ottey H. Plisson), I: 21-30. Eraul, Lieja. .

Shott, M.

1989 Diversity, Organization and Behavior in the Material Record: ethnographic and Archaeological Examples. Current Anthropology 30:283-315.

1997. Stones and shafts redux: the metric discrimination of chipped-stone dart and arrow points. American Antiquity 62:86-101.

Silvestre, R.

2004 Análisis de rastros de uso en lascas de filo natural del sitio arqueológico Anahí. Aproximaciones Contemporáneas a la Arqueología Pampeana. Perspectivas Teóricas, Metodológicas, Analíticas y Casos de Estudio (ed. por G. Martínez, M. A. Gutierrez, R. Curtoni, M. Berón y P. Madrid), pp.183-201. Facultad de Ciencias Sociales, UNCPBA Olavarría..

2010 Análisis funcional de artefactos líticos del Humedal del Paraná Inferior: El sitio Túmulo de Campana como caso de estudio. Tesis de Licenciatura, Buenos Aires, Facultad de Filosofía y Letras, UBA. 148 p.

2011a La tecnología lítica en el Sector Centro-Oriental de la Región Pampeana. Trabajo presentado en el VI CARPA. La Plata, 20-23 de septiembre 2011.

2011b Estrategias Tecnológicas de Grupos Guaraníes Prehistóricos: el sitio A Fredes como caso de estudio. Humedal del Paraná Inferior, Argentina. Trabajo enviado para su evaluación en Actas del $1^{\circ}$ Congreso Internacional de Arqueología de la Cuenca del Plata. Buenos Aires, 11-16 de abril 2011.

Smith, M. J., M. B. Brickley y S. L. Leach

2007 Experimental evidence for lithic projectile injuries: improving identification of an under-recognised phenomenon. Journal of Archaeological Science 34: 540-553.

Suárez R,

2009 Unifacial Fishtail Points and Considerations about the Archaeological Record of South American Paleoamericans. Current Research in the Pleistocene 26:12-15.

Tankersley, K. B.

1994 Clovis Mastic and its Hafting Implications. Journal of Arcaheological Science 21: 117-124.

Thomas, D. H.

1978 Arrowheads and Atlatl Darts: How the Stones Got the Shaft. American Antiquity 43 (3): 461-472.

Torres, L.M.

1911 Los Primitivos Habitantes del Delta del Paraná. Universidad Nacional de La Plata-Biblioteca Centenaria, La Plata.

Tyzzer, E. E.

1936 The "Simple Bone Point" of the Shell-Heaps of the Northeastern Algonkian Area and Its Probable Significance. American Antiquity 1 (4): 261-279. 
Vega, J. J. y R. E. Andrade

2004 «Infierno»: Resultados del análisis realizado sobre el material lítico recuperado en el sitio «Pingüino», departamento de Río Negro, República Oriental del Uruguay. X Congreso Uruguayo de Arqueología: La Arqueología Uruguaya ante los desafios del nuevo siglo (ed. por L. Beovide, I. Barreto y C. Curbelo). CD-ROM Multimedia Didáctico. Montevideo, Uruguay

Woodburn, J.

1980 Hunters and gatherers today and reconstruction of the past. Soviet and Western Anthropology (ed. por E. Gellner), pp. 95-117. Duckworth, London. 